\title{
Topology optimization using the lattice Boltzmann method incorporating level set boundary expressions
}

\section{AUTHOR(S):}

Yaji, Kentaro; Yamada, Takayuki; Yoshino, Masato; Matsumoto, Toshiro; Izui, Kazuhiro; Nishiwaki, Shinji

\section{CITATION:}

Yaji, Kentaro ... [et al]. Topology optimization using the lattice Boltzmann method incorporating level set boundary expressions. Journal of Computational Physics 2014, 274: $158-181$

\section{ISSUE DATE:}

2014-10

URL:

http://hdl.handle.net/2433/189430

\section{RIGHT:}

(C) 2014 Elsevier Inc:; This is not the published version. Please cite only the published version.; この論文は出版社版でありません。引用の際に は出版社版をご確認ご利用ください。 


\title{
Topology optimization using the lattice Boltzmann method incorporating level set boundary expressions
}

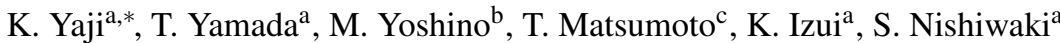 \\ ${ }^{a}$ Department of Mechanical Engineering and Science, Graduate School of Engineering, Kyoto University, Kyoto 615-8540, Japan \\ ${ }^{b}$ Department of Mechanical Systems Engineering, Faculty of Engineering, Shinshu University, Nagano 380-8553, Japan \\ ${ }^{c}$ Department of Mechanical Engineering and Science, Graduate School of Engineering, Nagoya University, Aichi 464-8603, Japan
}

\begin{abstract}
This paper presents a topology optimization method for fluid dynamics problems, based on the level set method and using the lattice Boltzmann method (LBM). In this optimization method, the optimization problems are formulated based on the original Boltzmann equation, and the design sensitivities are precisely obtained without the time-consuming numerical operations encountered when dealing with a large-scale asymmetric matrix, in contrast to previous research in which the LBM uses the lattice Boltzmann equation (LBE) for the formulations of optimization problems and the derivation of their adjoint equations. That is, we newly derive sensitivity formulations from the original Boltzmann equation, not the LBE that can be said to be an approximated equation, and these formulations yield strictly correct sensitivities that are error free. Based on the above formulations, we construct a level set-based topology optimization method incorporating a fictitious interface energy for the design of a fluid channel that minimizes flow friction. Furthermore, two- and three-dimensional numerical examples are provided to confirm the validity and utility of the presented method.
\end{abstract}

Keywords: Topology Optimization, Level Set Method, Phase Field Method, Lattice Boltzmann Method, Boltzmann Equation, Adjoint Variable Method

\section{Introduction}

This paper presents a new topology optimization method for fluid dynamics problems, based on the level set method and using the lattice Boltzmann method (LBM). The novel aspect of the presented method is the sensitivity formulations that are formulated based on the original Boltzmann equation, so that the design sensitivities can be precisely derived without the use of matrix operations.

Structural optimization is a methodology to obtain an optimal solution using a physical numerical model based on mathematical optimization theory. Since this methodology can obtain high performance structures using structural optimization techniques, it is very attractive, even in the field of fluid mechanics. In 1973, Pironneau [1] pioneered a structural optimization method for fluid dynamics problems, and obtained minimum drag wing profiles under Stokes flow. Considerable research has been carried out since then and a number of structural optimization methods applicable to fluid dynamics problems have been proposed [2-11]. However, since the above research was based on shape optimization, the feasible design modifications only consisted in the adjustment of the boundaries of selected parts to the fluid domain.

In contrast to shape optimization, where only the boundary of the design domain is optimized, topology optimization allows the creation of new holes and alteration of the connectivity of the design domain during the optimization process. Topology optimization has been extensively applied to a variety of structural optimization problems such as stiffness maximization problems [12,13], eigenfrequency problems [14-16], thermal problems [17, 18], and fluid

\footnotetext{
${ }^{*}$ Corresponding author

Email address: yaji.kentarou.74v@st.kyoto-u.ac.jp (K. Yaji )
} 
dynamics problems $[19,20]$, after Bendsøe and Kikuchi first proposed the so-called homogenization design method [12]. The basic concepts of topology optimization consist of (1) the extension of a design domain to a fixed design domain, and (2) replacement of the optimization problem with a material distribution problem, using the characteristic function originally presented in the papers of Murat and Tartar [21]. A homogenization method is utilized to deal with the extreme discontinuity of the material distribution in the design domain, and to provide the material properties viewed in a global sense as homogenized properties. This homogenization design method has been applied to a variety of design problems, and the density approach [22] is another currently used topology optimization method. The basic idea of the density approach is the use of a fictitious isotropic material whose elasticity tensor is assumed to be a function of penalized material density, expressed as an exponent parameter. In addition, topology optimization based on level set [23-25], phase field [26-28], and evolutionary [29] approaches have been proposed, and these methodologies are precisely categorized in a review paper [30].

Based on the density approach, Borrvall and Petersson [19] first proposed a topology optimization method for minimum power dissipation in a Stokes flow problem, where the material distribution in the fixed design domain is expressed as either the presence of fluid or an impermeable solid domain. Since the feasible design modifications pertain to adjustments of the material porosity in the fixed design domain, the no-slip boundary condition along the fluid-solid interface can be implicitly satisfied. In research based on this methodology, Aage et al. [31] proposed a topology optimization method for large-scale Stokes flow problems. Olesen et al. [20] proposed a topology optimization method using the steady-state Navier-Stokes (NS) equations for incompressible fluids, and introduced a numerical implementation scheme using commercial software. Deng et al. [32], and Kreissl et al. [33], proposed a topology optimization method using the unsteady NS equations for incompressible fluids. On the other hand, Kondoh et al. [34] obtained optimal body shapes in NS flows for drag minimization and lift maximization problems by introducing a new types of objective function. Furthermore, using Borrvall and Petersson's methodology as a basis, considerable research has been carried out to develop engineering applications for fluidic devices [35-37]. In addition, multiphysics topology optimization methods have been proposed to deal with fluid-structure [38], fluid-electric [39], fluid-thermal [40, 41], and electro-fluid-thermal-compliant [42] problems.

However, all of the above-mentioned research encounters the problem of so-called grayscales, regions of intermediate density that are allowed to exist in the optimal configurations. In such cases, the no-slip boundary condition is incompletely satisfied, since a specific boundary along the solid-fluid interface does not exist in grayscale regions. Guest and Prévost [43] employed their nodal design variable method, a kind of penalization scheme, to eliminate grayscales in topology optimization for minimum power dissipation problems under Stokes flow, but such filtering schemes crucially depend on artificial parameters that lack rational guidelines for determining appropriate $a$ priori parameter values.

A different approach is used in level set-based structural optimization methods that have been proposed as a new type of structural optimization method [23-25, 44-47]. Such methods implicitly represent target structural configurations using the iso-surface of the level set function $[48,49]$, which is a scalar function, and the outlines of target structures are changed by updating the level set function during the optimization process. In level set-based structural optimization methods, the obtained optimal configurations are free from grayscales, since the structural boundaries are represented by the iso-surface of the level set function. Challis and Guest [50] proposed a level set-based structural optimization method for minimum power dissipation problems under Stokes flow, and examined the same design problems as those treated in previous research using the density approach [19, 43]. Duan et al. [51], Zhou et al. [52], and Duan et al. [53] proposed a level set-based structural optimization method for steady-state NS flow problems, and Deng et al. [54] extended it to unsteady NS flow optimization problems. However, since these level set-based structural optimization methods are essentially based on shape optimization methods that obtain optimal configurations by moving structural boundaries, they do not allow topological changes such as the creation of new holes during optimization procedure. Allaire et al. [55] introduced the bubble method [56] to a level set-based shape optimization method using topological derivatives $[57,58]$ in order to provide for the possibility of topological changes. However, the setting of parameter values to facilitate the introduction of holes during the optimization process was difficult, and the obtained optimal configurations often showed dependency on initial parameter values. As a radical way of solving the above problem, Yamada et al. [59] proposed a new level set-based approach, based on the concept of the phase field method and the use of a reaction-diffusion equation for updating the level set function, which allows topological changes during optimization procedure and exhibits minimal dependency on initial configuration or mesh size. This level set-based approach has been applied to a range of physical problems: stiffness maximization problems [59, 60], 
eigenfrequency problems [59], thermal problems [61, 62], electromagnetic problems [63-66], and acoustic problems [67]. In addition, Talischi and Glaucio [68] have recently proposed a method similar to Yamada et al's approach, for stiffness maximization problems.

In structural optimization methods for fluid dynamics problems, reduction of flow field computational cost is a major factor when seeking to maintain practical total optimization times, since most numerical schemes for obtaining solutions to NS equations for incompressible fluids include an iterative computation of a massive system of linear equations, which is related to the integration of the Poisson equation for the pressure field. That is, structural optimization methods for large-scale flow problems typically incur great computational cost to obtain optimal configurations. Similarly, since most previous research on structural optimization methods for fluid dynamics problems employ the finite element method (FEM) to obtain solutions of the incompressible NS equations, the scale of feasible computational space has been very limited. Consequently, most numerical examples in previous research deal with two-dimensional cases, and those that do address three-dimensional cases have a relatively small number of finite elements, such as the 47,151 elements used for the design of a flow channel in Aage et al. [31]. To deal with large-scale flow problems in structural optimization problems, these computational obstacles must be overcome.

On the other hand, the lattice Boltzmann method (LBM) [69-74] has attracted attention as an alternative and promising numerical scheme for obtaining solutions to the NS equations for incompressible fluids, without dealing with the Poisson equation for the pressure field. In the LBM, the velocity distribution functions and a set of discrete fictitious particle velocities are explicitly computed, using the so-called lattice Boltzmann equation (LBE) that tracks the time evolution of the velocity distribution functions. The macroscopic variables such as the velocity and pressure are obtained by the moments of the velocity distribution functions, and satisfy the fluid dynamics conservation laws for mass, momentum, and energy. Considerable research has dealt with the construction of a mathematical theory that incorporates the above laws, such as the Chapman-Enskog expansion [72], the S-expansion of asymptotic theory $[75,76]$, and others $[77,78]$. Because the LBM guarantees the conservation of mass, momentum, and energy, it can be applied to multiphase flows [79-82] and the interface can be represented clearly, without any special treatments. Thus, with the LBM, the interface does not have to be explicitly tracked during the numerical computation. In addition, taking advantage of the fact that the algorithm is simple, computationally efficient, as well as highly scalable for parallel processing, many researchers have investigated complex and large-scale flows such as porous flows [8386] and turbulent flows [87-91], and an immersed boundary method based on the LBM [92-95] has recently attracted attention in moving body problems.

The LBM is therefore extremely useful when working with complex and large-scale flow problems and can be successfully applied to structural optimization problems. In a pioneering study by Pingen et al. [96], a topology optimization methodology using the LBM was proposed and optimal configurations similar to those of a previous approach [19] proposed by Borrvall and Petersson using the FEM were obtained. Based on this pioneering study using the LBM, Pingen et al. [97] and Kreissl et al. [98] proposed a level-set based structural optimization method using the LBM for a flow channel design problem. Pingen and Maute [99] dealt with non-Newtonian flows to represent the viscosity of blood in their design model of a flow channel. Kreissl et al. [100] proposed a topology optimization method for a fluid-structure interaction problem for micro-channel devices. In addition, Makhija et al. [101] proposed a topology optimization method using the LBM for a mixture efficiency maximization problem under multi-component flow.

However, in the above-mentioned methodologies that employ the LBM, the design sensitivities cannot be treated precisely, since the LBE, which can be said to be an approximated equation, is used for the formulations of the optimization problems and the derivation of their adjoint equation. Furthermore, a large-scale asymmetric matrix must be dealt with to obtain the design sensitivities in each iteration of the optimization process [102]. Thus, timeconsuming numerical operations are required, and the advantages of the LBM, such as its algorithmic simplicity and computational efficiency, cannot be applied to the process of solving the adjoint equation.

In this paper, we construct a topology optimization method based on level set boundary expressions [59] using the LBM, in which the optimization problems are formulated based on the Boltzmann equation, not the LBE, and these formulations yield strictly correct sensitivities without errors. Furthermore, the adjoint equation we use has an advantage, namely that a novel discretization strategy similar to the LBM can lead to algorithms that are as efficient as those of the LBE, due to similar locality properties. That is, the design sensitivities can be obtained during the optimization process without the use of matrix operations. This novel strategy of sensitivity formulation was first proposed by Krause et al. [103], and has not yet been applied to structural optimization problems. Therefore, we 
must confirm that it is appropriate for use in a topology optimization method that employs the LBM. Based on the above formulation, we construct a level set-based topology optimization method for fluid dynamics problems that incorporates the fictitious interface energy proposed by Yamada et al. [59].

In the following sections, the Boltzmann equation and the LBE are first discussed as core concepts of the LBM. Next, the proposed level set-based topology optimization method is described and the optimization problem is formulated using the Boltzmann equation. The numerical implementation and optimization algorithm are then described and, finally, we introduce two- and three-dimensional numerical examples to validate the utility of the topology optimization method.

\section{Governing equation}

The LBM [71-74] is a new numerical scheme in computational fluid dynamics for simulating fluid flows and modeling physics in fluids. The basic concept of the LBM is to construct kinetic models that incorporate the essential physics of microscopic processes so that the macroscopic properties are correctly modeled in the mesoscopic equations. The LBM expresses the fluid regime via an aggregation of fictitious particles, and makes it possible to obtain macroscopic values such as the fluid density and the fluid velocity from the moments of the velocity distribution functions that express the distribution state of the particles.

In this section, we discuss the concept of the LBM, which is applied to an incompressible viscous fluid in an isothermal field. Hereafter, as shown in Appendix A, we use non-dimensional variables defined by a characteristic length $L$, a characteristic particle speed $c$, a characteristic time-scale $\tilde{t}=L / U$ where $U$ is a characteristic flow speed, a reference order parameter $f_{0}$, a reference density $\rho_{0}$, and a reference temperature $T_{0}$.

\subsection{Boltzmann equation}

The Boltzmann equation is a type of kinetic equation, and is often used for analyzing transport phenomena such as thermal conduction and diffusion, based on the kinetic theory of gases. The LBM is a numerical scheme using a discretized Boltzmann equation, the so-called lattice Boltzmann equation (LBE), that can represent macroscopic quantities such as fluid velocity and pressure by incorporating velocity distribution functions. In this study, the Boltzmann equation is used in the formulation of the optimization problems so that the design sensitivities can be efficiently derived. Based on these concepts, we now discuss the Boltzmann equation, which can be represented as follows, using the velocity distribution function $f=f(\boldsymbol{x}, t, \boldsymbol{\xi})$ :

$$
\operatorname{Sh} \frac{\partial f}{\partial t}+\boldsymbol{\xi} \cdot \nabla f=Q(f) \quad \text { in } \quad I \times \Omega \times \Xi
$$

where $\mathrm{Sh}=L /(\tilde{t} c)$ is a non-dimensional parameter (the Strouhal number), $t \in I\left[t_{0}, t_{1}\right) \subseteq \mathbb{R}_{\geqslant 0}$ represents the time, $\boldsymbol{x} \in \Omega \subset \mathbb{R}^{d}$ and $\boldsymbol{\xi} \in \Xi\left(=\mathbb{R}^{d}\right)$ represent the gas particle position and velocity, respectively, and $Q$, a so-called a collision operator, expresses the effect of collision between the fictitious particles. Superscript $d$ in the above represents the number of spatial dimensions, which is either 2 or 3 . For the sake of simplicity and without losing generality, we use the Bhatnagar-Gross-Krook (BGK) collision model [104] as follows:

$$
Q(f)=-\frac{1}{\tau_{\mathrm{B}}}\left(f-f^{\mathrm{eq}}\right),
$$

where $\tau_{\mathrm{B}}$ is the dimensionless relaxation time that expresses the average time until the next collision. $f^{\text {eq }}$ is a Maxwell distribution as a local equilibrium solution of the Boltzmann equation,

$$
f^{\mathrm{eq}}=\frac{\rho}{T^{d / 2}} \exp \left(-\frac{|\boldsymbol{\xi}-\boldsymbol{u}|^{2}}{T}\right),
$$

where $\rho$ and $\boldsymbol{u}$ represent the fluid density and velocity, respectively. Here, $T$ is the temperature that assumes a constant value in the isothermal condition. Based on kinetic theory, the macroscopic variables in the flow field, i.e., the density $\rho$, and the fluid velocity $\boldsymbol{u}$ can be derived by the moments of the velocity distribution function $f$ with respect to the velocity field $\Xi$ :

$$
\rho=\int_{\Xi} f \mathrm{~d} \boldsymbol{\xi}, \quad \boldsymbol{u}=\frac{1}{\rho} \int_{\Xi} \boldsymbol{\xi} f \mathrm{~d} \boldsymbol{\xi} .
$$




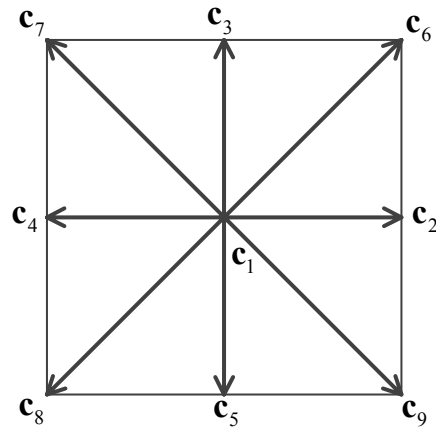

(a) 2D9V model

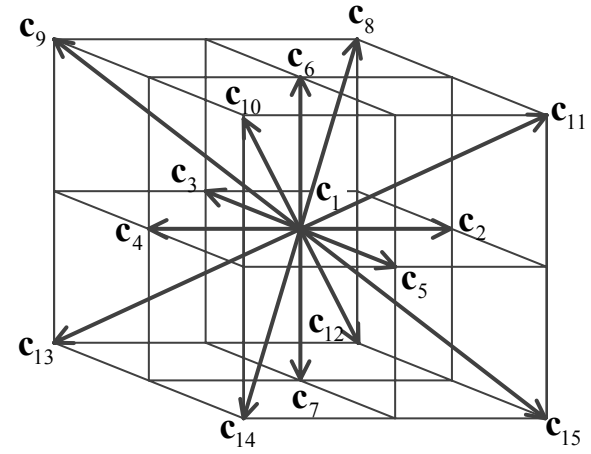

(b) 3D15V model

Figure 1: Particle velocity vectors $\boldsymbol{c}_{i}$ of (a) nine-velocity model and (b) fifteen-velocity model

\subsection{Lattice Boltzmann equation}

Based on the Boltzmann equation (1), we now discuss the lattice Boltzmann equation that is used in the LBM. The basic idea of the LBM is the introduction of a finite number of particle velocities that replaces the infinite set of particle velocities $\boldsymbol{\xi}$ in Eq. (1) This allows macroscopic quantities such as flow velocity and pressure, derived by the moments of a finite number of velocity distribution functions, to be obtained as solutions of the Navier-Stokes equations.

Consider a modeled gas composed of identical particles whose velocities are restricted to a finite set of $q$ vectors, $\boldsymbol{c}_{1}, \boldsymbol{c}_{2}, \cdots, \boldsymbol{c}_{q} \in \Xi^{h}$, while the above mentioned Boltzmann equation (1) treats the infinite set of $\boldsymbol{\xi}$. That is, the Boltzmann equation using the BGK model, where the particle velocities belong to the discrete space $\Xi^{h}$, is given as the following discrete Boltzmann equation [105] for the velocity distribution functions $f_{i}(\boldsymbol{x}, t)=f\left(\boldsymbol{x}, t, \boldsymbol{c}_{i}\right)$ :

$$
\operatorname{Sh} \frac{\partial f_{i}}{\partial t}+\boldsymbol{c}_{i} \cdot \nabla f_{i}=-\frac{1}{\tau_{\mathrm{B}}}\left(f_{i}-f_{i}^{\mathrm{eq}}\right) \quad \text { in } \quad I \times \Omega \times \Xi^{h} .
$$

The value of $q$ is defined differently in various lattice gas models [73]. In the two-dimensional case, we employ the nine-velocity (2D9V) model, which has the following velocity vectors:

$$
\begin{aligned}
& {\left[\boldsymbol{c}_{1}, \boldsymbol{c}_{2}, \boldsymbol{c}_{3}, \boldsymbol{c}_{4}, \boldsymbol{c}_{5}, \boldsymbol{c}_{6}, \boldsymbol{c}_{7}, \boldsymbol{c}_{8}, \boldsymbol{c}_{9}\right]} \\
& =\left[\begin{array}{rrrrrrrrr}
0 & 1 & 0 & -1 & 0 & 1 & -1 & -1 & 1 \\
0 & 0 & 1 & 0 & -1 & 1 & 1 & -1 & -1
\end{array}\right] .
\end{aligned}
$$

In the three-dimensional case, we employ the fifteen-velocity (3D15V) model, which has the following velocity vectors:

$$
\begin{aligned}
& {\left[\boldsymbol{c}_{1}, \boldsymbol{c}_{2}, \boldsymbol{c}_{3}, \boldsymbol{c}_{4}, \boldsymbol{c}_{5}, \boldsymbol{c}_{6}, \boldsymbol{c}_{7}, \boldsymbol{c}_{8}, \boldsymbol{c}_{9}, \boldsymbol{c}_{10}, \boldsymbol{c}_{11}, \boldsymbol{c}_{12}, \boldsymbol{c}_{13}, \boldsymbol{c}_{14}, \boldsymbol{c}_{15}\right]} \\
& =\left[\begin{array}{rrrrrrrrrrrrrrr}
0 & 1 & 0 & -1 & 0 & 0 & 0 & 1 & -1 & -1 & 1 & 1 & -1 & -1 & 1 \\
0 & 0 & 1 & 0 & -1 & 0 & 0 & 1 & 1 & -1 & -1 & 1 & 1 & -1 & -1 \\
0 & 0 & 0 & 0 & 0 & 1 & -1 & 1 & 1 & 1 & 1 & -1 & -1 & -1 & -1
\end{array}\right] .
\end{aligned}
$$

These velocity models are shown in Fig. 1. In Eq. (5), discrete local equilibrium distribution function $f_{i}^{\text {eq }}$ is obtained by the Maxwell distribution (3), which can be approximated as a Taylor expansion if the velocity is small or has a very low Mach number, which implies that the characteristic speed $U$ is much smaller than the speed of sound. Thus, the discrete equilibrium distribution function $f_{i}^{\mathrm{eq}}$ up to $O\left(U^{2}\right)$ is represented as follows [72]:

$$
f_{i}^{\mathrm{eq}}=w_{i} \rho\left\{1+3 \boldsymbol{c}_{i} \cdot \boldsymbol{u}+\frac{9}{2}\left(\boldsymbol{c}_{i} \cdot \boldsymbol{u}\right)^{2}-\frac{3}{2} \boldsymbol{u} \cdot \boldsymbol{u}\right\} .
$$


For the 2D9V model, the weight $w_{i}$ is defined so that $w_{1}=4 / 9, w_{2}=w_{3}=w_{4}=w_{5}=1 / 9, w_{6}=w_{7}=w_{8}=w_{9}=1 / 36$, and for the $3 \mathrm{D} 15 \mathrm{~V}$ model, the weight $w_{i}$ is defined so that $w_{1}=2 / 9, w_{2}=w_{3}=\cdots=w_{7}=1 / 9, w_{8}=w_{9}=\cdots=w_{15}=$ $1 / 72$. The density $\rho$, and the fluid velocity $\boldsymbol{u}$ are obtained from the following moments of the velocity distribution functions:

$$
\rho=\sum_{i=1}^{q} f_{i}, \quad \boldsymbol{u}=\frac{1}{\rho} \sum_{i=1}^{q} \boldsymbol{c}_{i} f_{i} .
$$

In using the $2 \mathrm{D} 9 \mathrm{~V}$ or $3 \mathrm{D} 15 \mathrm{~V}$ models, the pressure $p$ is represented as follows:

$$
p=\frac{\rho}{3}
$$

In the LBM, physical space is divided into a square or cubic lattice with spacing $\Delta x \in \Omega^{h} \subset \mathbb{R}_{>0}$, and time steps $\Delta t \in I^{h}$, where the discrete time interval $I^{h}:=\left\{t \in I: t=t_{0}+k \Delta t, k \in \mathbb{N}\right\}$ is employed when formulating the LBE. Using $\Delta x$ and $\Delta t$, the discrete Boltzmann equation (5) for three-dimensional cases, in which the particle velocities are defined as $c_{i}=\left[c_{i x}, c_{i y}, c_{i z}\right]$, can be discretized as follows:

$$
\begin{aligned}
& \frac{f_{i}(x, y, z, t+\Delta t)-f_{i}(x, y, z, t)}{\Delta t} \\
& \quad+\frac{c_{i x}}{\operatorname{Sh}} \frac{f_{i}\left(x+c_{i x} \Delta x, y+c_{i y} \Delta x, z+c_{i z} \Delta x, t+\Delta t\right)-f_{i}\left(x, y+c_{i y} \Delta x, z+c_{i z} \Delta x, t+\Delta t\right)}{c_{i x} \Delta x} \\
& \quad+\frac{c_{i y}}{\operatorname{Sh}} \frac{f_{i}\left(x, y+c_{i y} \Delta x, z+c_{i z} \Delta x, t+\Delta t\right)-f_{i}\left(x, y, z+c_{i z} \Delta x, t+\Delta t\right)}{c_{i y} \Delta x} \\
& \quad+\frac{c_{i z}}{\operatorname{Sh}} \frac{f_{i}\left(x, y, z+c_{i z} \Delta x, t+\Delta t\right)-f_{i}(x, y, z, t+\Delta t)}{c_{i z} \Delta x} \\
& =-\frac{1}{\tau_{\mathrm{B}} \operatorname{Sh}}\left\{f_{i}(x, y, z, t)-f_{i}^{\mathrm{eq}}(x, y, z, t)\right\} .
\end{aligned}
$$

Here, we choose $\Delta t=\operatorname{Sh} \Delta x$ so that particles at any lattice node can propagate to any immediately neighboring lattice node during each time step. This is possible because $\Delta t=\operatorname{Sh} \Delta x$ in non-dimensional form leads to $\Delta \bar{t} / \tilde{t}=$ $L /(\tilde{t} c) \cdot(\Delta \bar{x} / L)$, i.e., $\Delta \bar{t}=\Delta \bar{x} / c$ in dimensional form. In addition, we note that the above discretization technique is different from that usually used, in which the first-order upwind scheme is applied to obtain the LBE from the original Boltzmann equation. The use of the different notation scheme allows very similar presentations of the discretization for both the adjoint equation in Eq. (48), discussed in section 4.2, and the Boltzmann equation.

Consequently, we obtain the following LBE:

$$
f_{i}\left(\boldsymbol{x}+\boldsymbol{c}_{i} \Delta x, t+\Delta t\right)=f_{i}(\boldsymbol{x}, t)-\frac{1}{\tau_{\mathrm{B}}}\left\{f_{i}(\boldsymbol{x}, t)-f_{i}^{\mathrm{eq}}(\boldsymbol{x}, t)\right\}, \quad \text { in } I^{h} \times \Omega^{h} \times \Xi^{h} .
$$

We note that Eq. (12) can also be formulated for two-dimensional cases if particle velocities $\boldsymbol{c}_{i}=\left[c_{i x}, c_{i y}\right]$ are used.

\section{Optimization problem}

In this section, using the LBM, we formulate the topology optimization problem incorporating level set boundary expressions for the design problem dealing with the isothermal viscous fluid. To derive the design sensitivities, we use the adjoint variable method [106] and employ the Boltzmann equation to obtain an adjoint equation that can be discretized so that the formulation resembles that of the LBE. Since the adjoint equation is also computed explicitly, as with the LBE, simple computation algorithms for the state and adjoint problems can be constructed. In the following, we formulate the optimization problem using the Boltzmann equation in order to exploit this advantage. 


\subsection{Topology optimization method}

Consider a structural optimization problem that determines the the boundary of the design domain $\Omega$ by minimizing or maximizing objective functions. The key idea of the topology optimization method is the introduction of a fixed design domain $D$ that includes the original design domain $\Omega$, and the utilization of the following characteristic function, $\chi_{\Omega}$, described in the papers of Murat and Tartar [21], Belyschko et al. [107], and Norato et al. [108]:

$$
\chi_{\Omega}(x)= \begin{cases}1 & \text { if } \quad x \in \Omega, \\ 0 & \text { if } \quad x \in D \backslash \Omega,\end{cases}
$$

where $\boldsymbol{x}$ represents a position in the fixed design domain $D$. Using this characteristic function, the original structural optimization problem is replaced with a material distribution problem in the fixed design domain $D$. Since this characteristic function can be highly discontinuous, i.e. resides in $L^{\infty}(D)$, some regularization or smoothing technique such as the homogenization method [109] must be introduced for the numerical treatment.

In such regularization techniques, the existence of grayscales is allowed in the optimal configurations. Although grayscales can be interpreted as being micro-porous in a physical sense, they are problematic in an engineering sense since such optimal configurations are difficult to interpret as practical designs that can be manufactured.

\subsection{Level set boundary expressions}

A different approach is used in level set-based structural optimization methods that have been proposed as a new type of structural optimization method [23-25, 44-47]. Such methods implicitly represent target structural configurations using the iso-surface of the level set function, which is a scalar function, and the outlines of target structures are changed by updating the level set function during the optimization process. In level set-based structural optimization methods, the obtained optimal configurations are free from grayscales, since the structural boundaries are represented as the iso-surface of the level set function. Using the level set method to represent the boundaries of the flow field, an optimal configuration that has clear boundaries between fluid and solid domains can be derived. Here, the level set function $\phi$ represents fluid and solid domains, so that an optimization problem formulated for a fluid dynamics problem can be considered, while the boundaries between these domains, $\partial \Omega$, are represented by the iso-surface of the level set function, as follows:

$$
\left\{\begin{array}{lll}
0<\phi(x) \leqslant 1 & \text { if } & x \in \Omega \backslash \partial \Omega, \\
\phi(x)=0 & \text { if } \quad x \in \partial \Omega \\
-1 \leqslant \phi(x)<0 & \text { if } \quad x \in D \backslash \Omega
\end{array}\right.
$$

where $\Omega$ represents the fluid domain, and $D \backslash \Omega$ represents the solid domain. As shown in Fig. 2, the fluid and solid domains are defined as the level set function assumes positive and negative values, respectively. Based on the previous study [59], we assume that the distribution of the level set function $\phi$ have the same property of distribution as the phase field variable in the phase field method. Therefore, the level set function is constrained to values lying between -1 and 1 in Eq. (14).

\subsection{Expression of fluid and solid domains using the level set function}

Topology optimization for a fluid problem deals with fluid and solid domains in the fixed design domain. In previous studies (e.g. [19, 43, 110]) using the FEM, the fixed design domain is expressed as either containing fluid or an impermeable solid. Since the feasible design modifications involve adjusting the material porosity in the fixed design domain, the no-slip boundary condition along the fluid-solid interface can be implicitly satisfied. Unfortunately, this assumption requires the use of a fictitious external force, based on a theoretical description of porosity, expressed as an additional term in the NS equation, and an appropriate parameter value for the porosity must be set by trial and error.

In this study, we utilize the characteristic of the Boltzmann equation to introduce a simple expression for the fluid and solid domains, using the above level set method. That is, the Maxwell distribution $f^{\text {eq }}$ in Eq. (3) is replaced with 


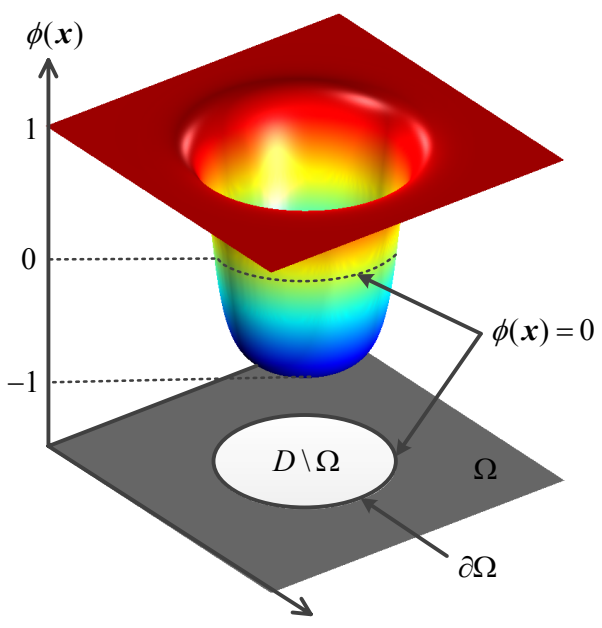

Figure 2: Fixed design domain $D$ and level set function $\phi$

the following extended Maxwell distribution $\hat{f}^{\mathrm{eq}}$ in order to extend the fluid domain $\Omega$ to the fixed design domain $D$, as follows:

$$
\hat{f}^{\mathrm{eq}}\left(\rho, \boldsymbol{u}, \chi_{\phi}\right)=\frac{\rho}{T^{d / 2}} \exp \left(-\frac{\left|\boldsymbol{\xi}-\chi_{\phi} \boldsymbol{u}\right|^{2}}{T}\right),
$$

where $\chi_{\phi}$ is a characteristic function that depends on the value of the level set function $\phi$, and represents the existence of the fluid domain $\Omega$ according to the following definition:

$$
\chi_{\phi}= \begin{cases}1 & \text { if } \quad \phi(x) \geqslant 0 \\ 0 & \text { if } \quad \phi(x)<0 .\end{cases}
$$

Since the Boltzmann equation is made dependent on the characteristic function $\chi_{\phi}$, the solid domain can be represented as the zero velocity field by using Eq. (15). Consequently, the space of the Boltzmann equation in Eq. (1) can be considered as $f \in I \times D \times \Xi$, which allows the flow regime to be represented in the fixed design domain $D$. Therefore, an optimal configuration can be obtained by controlling $\chi_{\phi}$, governed by the level set function $\phi$ as a design variable in the optimization problem.

\subsection{Extended Boltzmann equation and initial and boundary conditions}

Using the Boltzmann equation incorporating the extended Maxwell distribution $\hat{f}^{\mathrm{eq}}$ in Eq. (15), we formulate a topology optimization problem for the flow channel design problem, which requires the setting of appropriate initial and boundary conditions. Here, we consider the following formulation of the Boltzmann equation, and initial and boundary conditions:

$$
\begin{array}{ll}
\operatorname{Sh} \frac{\partial f}{\partial t}+\boldsymbol{\xi} \cdot \nabla f=-\frac{1}{\tau_{\mathrm{B}}}\left\{f-\hat{f}^{\mathrm{eq}}\left(\rho[f], \boldsymbol{u}[f], \chi_{\phi}\right)\right\} & \text { in } I \times D \times \Xi, \\
\left.f\right|_{t=t_{0}}=f^{0} & \text { in } D \times \Xi, \\
f(\boldsymbol{\xi})=f(-\boldsymbol{\xi}) & \text { in } I \times \Gamma_{\mathrm{w}} \times \Xi_{n} \cdot \boldsymbol{\xi}<0, \\
f=f^{\mathrm{eq}}\left(\rho[f], \boldsymbol{u}_{\text {in }}\right) & \text { in } I \times \Gamma_{\text {in }} \times \Xi, \\
f=f^{\mathrm{eq}}\left(\rho_{\text {out }}, \boldsymbol{u}[f]\right) & \text { in } I \times \Gamma_{\text {out }} \times \Xi,
\end{array}
$$

where Eq. (18) represents the initial condition of the expanded Boltzmann equation (17), and $f^{0} \in \mathbb{R}_{>0}$ is an initial value of the velocity distribution function. Equations (19)-(21) represent the boundary conditions. Eq. (19) is the 


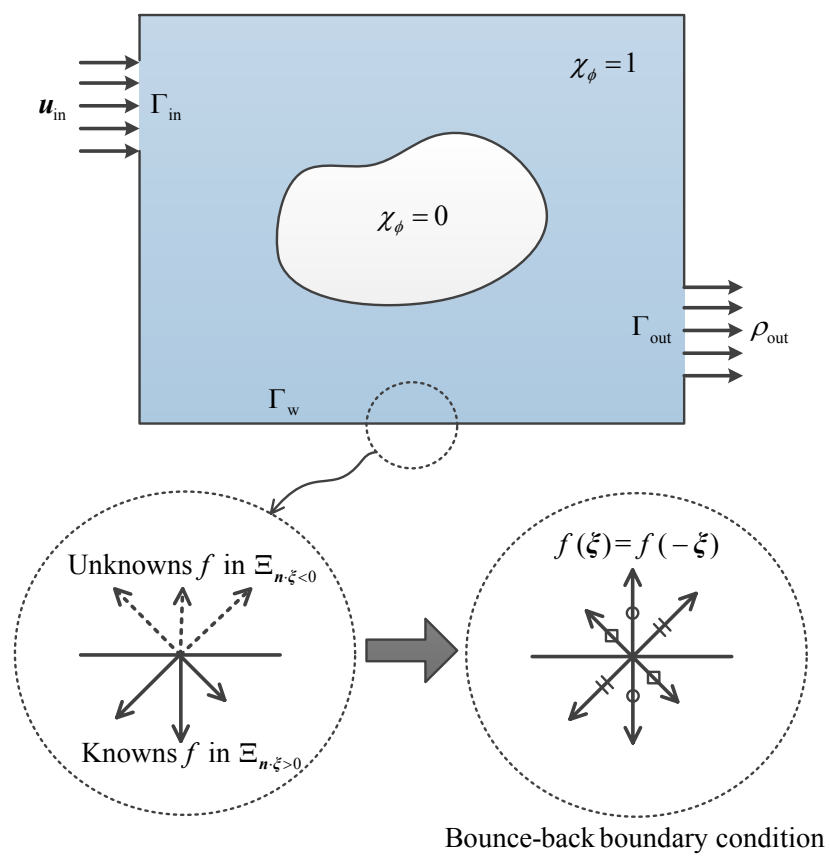

Figure 3: Design specifications for flow field governed by the Boltzmann equation

non-slip boundary on the wall, $\Gamma_{\mathrm{w}}$, called a bounce-back boundary condition [73] that is defined in the velocity space $\Xi_{n \cdot \xi<0}$ (where $\boldsymbol{n}$ is a normal vector on the boundary of the fixed design domain directed outward). Equations (20) and (21) represent the inlet and outlet boundary conditions, respectively, using a prescribed velocity $\boldsymbol{u}_{\text {in }} \in \mathbb{R}^{d}$ on inlet $\Gamma_{\text {in }}$, and density $\rho_{\text {out }} \in \mathbb{R}_{>0}$ on outlet $\Gamma_{\text {out }}$. The schematic of the flow channel problem based on the above formulation is shown in Fig. 3.

\subsection{Formulation of optimization problem}

Here, using the Boltzmann equation, an optimization problem is formulated with respect to a general form of objective functional $J$, which evaluates the performance of the flow channel optimization problem in the fixed design domain $D$ and its boundary $\Gamma_{\text {in }} \cup \Gamma_{\text {out }}$. However, we must note that, since the LBM is an explicit time evolution scheme for computing transient flows that requires sufficient computing time to represent a practical flow field, the objective functional $J$ should be evaluated only after a certain practical time interval has passed from the initial time step, $t_{0}$. Thus, we define the practical time interval as a steady-state flow condition where the appropriate time at which the objective functional can be evaluated is represented as $t_{1}$, and the new objective functional is represented as $J=\left.J\right|_{t=t_{1}}$. We use macroscopic variables (i.e., density $\rho$ and velocity $\boldsymbol{u}$ ), and formulate the optimization problem 
using the Boltzmann equation with respect to the objective functional $\left.J\right|_{t=t_{1}}$ by integrating $A$ and $a$, as follows:

$$
\begin{aligned}
\left.\inf _{\phi} J\right|_{t=t_{1}} & =\int_{D} A(\rho[f], \boldsymbol{u}[f]) \mathrm{d} \Omega+\int_{\Gamma_{\text {in }} \cup \Gamma_{\text {out }}} a(\rho[f], \boldsymbol{u}[f]) \mathrm{d} \Gamma, \\
\text { s.t. } \quad V & =\int_{D} \chi_{\phi} \mathrm{d} \Omega-V_{\max } \leqslant 0, \\
G & =\int_{I} \int_{D} \int_{\Xi} g\left\{\operatorname{Sh} \frac{\partial f}{\partial t}+\boldsymbol{\xi} \cdot \nabla f+\frac{1}{\tau_{\mathrm{B}}}\left(f-\hat{f}^{\mathrm{eq}}\right)\right\} \mathrm{d} \boldsymbol{\xi} \mathrm{d} \Omega \mathrm{d} t=0, \\
I & =\int_{D} \int_{\Xi} g\left(\left.f\right|_{t=t_{0}}-f^{0}\right) \mathrm{d} \boldsymbol{\xi} \mathrm{d} \Omega \mathrm{d} t=0, \\
B_{1} & =\int_{I} \int_{\Gamma_{\mathrm{w}}} \int_{\Xi} g\{f(\boldsymbol{\xi})-f(-\boldsymbol{\xi})\} \mathrm{d} \boldsymbol{\xi} \mathrm{d} \Gamma \mathrm{d} t=0, \\
B_{2} & =\int_{I} \int_{\Gamma_{\text {in }}} \int_{\Xi} g\left\{f-f^{\mathrm{eq}}\left(\rho[f], \boldsymbol{u}_{\text {in }}\right)\right\} \mathrm{d} \boldsymbol{\xi} \mathrm{d} \Gamma \mathrm{d} t=0, \\
B_{3} & =\int_{I} \int_{\Gamma_{\text {out }}} \int_{\Xi} g\left\{f-f^{\mathrm{eq}}\left(\rho_{\text {out }}, \boldsymbol{u}[f]\right)\right\} \mathrm{d} \boldsymbol{\xi} \mathrm{d} \Gamma \mathrm{d} t=0,
\end{aligned}
$$

where $V$ is a volume constraint that prescribes the limit quantity $V_{\max } \in \mathbb{R}_{\geqslant 0}$ of the fluid domain, and $G, I$, and $B_{n}$ $(n=1,2,3)$ are respectively the weak forms of Eqs. (17)-(21) using the test function $g \in H^{1}(I \times D \times \Xi)$. In the above optimization problem, since the objective functional $\left.J\right|_{t=t_{1}}$ is evaluated under the steady-state condition, we define $t_{1}$ as the iteration time that satisfies the criterion of the steady-state flow condition. The specific definition of this criterion is described in the following section.

Since the characteristic function $\chi_{\phi}$ allows discontinuity in infinitesimal intervals throughout the fixed design domain $D$, the above optimization problem formulation is ill-posed. To regularize the optimization problem, an extended objective functional $J_{R}$ is defined as follows, based on the Tikhonov regularization scheme [111]:

$$
J_{R}:=J+R_{\tau},
$$

where the regularization term $R_{\tau}$ is defined as follows, using a regularization coefficient, $\tau \in \mathbb{R}_{>0}$ :

$$
R_{\tau}:=\int_{D} \frac{1}{2} \tau|\nabla \phi|^{2} d \Omega
$$

Consequently, the regularized optimization problem is formulated as

$$
\begin{array}{cl}
\underset{\phi}{\inf } & J_{R}=J+R_{\tau}, \\
\text { s.t. } & V \leqslant 0, \\
& G=0, I=0, B_{n}=0 .
\end{array}
$$

The above regularized optimization problem is now replaced by an unconstrained problem, using Lagrange's method of undetermined multipliers.

$$
\inf _{\phi} \quad \bar{J}_{R}=J_{R}+\lambda V+G+I+B_{n},
$$

where $\bar{J}_{R}$ is the regularized Lagrangian, and $\lambda \in \mathbb{R}$ is the Lagrange multiplier. Based on the above formulation, the KKT (Karush-Kuhn-Tucker) conditions of this optimization problem are formulated as follows:

$$
\delta_{\phi} \bar{J}_{R}=0, \quad G=0, \quad I=0, \quad B_{n}=0, \quad \lambda V=0, \quad \lambda \geqslant 0, \quad V \leqslant 0,
$$

where the notation $\delta_{\phi} \bar{J}_{R}:=\left\langle\vec{J}_{R}, \delta \phi\right\rangle$ represents the first variation of the regularized Lagrangian $\bar{J}_{R}$, using the Gâteaux derivative of $\bar{J}_{R}$ with respect to $\phi$ in the direction of $\delta \phi$. Level set functions that satisfy the above KKT conditions are candidate solutions of the level set function that describe optimal configurations. However, it is not easy to directly 
obtain these optimal solutions, so the optimization problem is replaced by the following time evolution problem of the level set function $\phi=\phi(\varsigma, x)$ by introducing a fictitious time, $\varsigma \in \Psi\left[\varsigma_{0}, \varsigma_{1}\right) \subseteq \mathbb{R}_{\geqslant 0}$, as follows:

$$
\begin{array}{ll}
\frac{\partial \phi}{\partial \varsigma}=-K \vec{J}_{R}=-K\left(\vec{J}^{\prime}-\tau \nabla^{2} \phi\right) & \text { in } \Psi \times D, \\
\phi=\phi^{0} & \text { in } \quad D, \\
\frac{\partial \phi}{\partial \boldsymbol{n}}=\mathbf{0} & \text { in } \Psi \times\left(\Gamma_{\text {in }} \cup \Gamma_{\text {out }}\right), \\
\phi=-1 & \text { in } \Psi \times \Gamma_{\mathrm{w}},
\end{array}
$$

where $K \in \mathbb{R}_{>0}$ is a constant of proportionality, and $\phi^{0} \in \mathbb{R}$ is the initial value of the level set function. Equations (37), and (38) and (39), respectively represent the initial and boundary conditions of Eq. (36). The results of the time evolution formulation are assumed to be proportional to the gradient of Lagrangian $\bar{J}_{R}$ with respect to the level set function $\phi$. The optimal configuration can be obtained by solving the above time evolution problem.

\subsection{Sensitivity analysis}

A key component of topology optimization method using the LBM is the use of the adjoint variable method [106] for derivation of the design sensitivities. Here, we discuss the sensitivity analysis based on the adjoint variable method, which is used in the topology optimization that employs the LBM. To clarify the difference between a conventional approach and our newly proposed method, we briefly introduce the method proposed by Pingen et al. [96, 112] for the formulation of the design sensitivities in an optimization problem using the LBE. We then discuss our approach in which the optimization problem is formulated using the original Boltzmann equation.

\subsubsection{Conventional sensitivity formulation using the $L B E$}

In previous research by Pingen et al. [96, 112], the optimization problem was formulated under a steady-state flow condition. Since the standard LBM is an explicit time evolution scheme for computing time-dependent flows, the flow needs to be advanced in time until convergence to a steady-state condition is satisfied. Thus, the LBE for steady-state flow can be represented as follows:

$$
\mathbf{R}(\mathbf{f}, \mathbf{p})=\mathbf{M}(\mathbf{f}, \mathbf{p})-\mathbf{f}=\mathbf{0},
$$

where $\mathbf{R}$ represents the residual vector, and $\mathbf{f}$ and $\mathbf{p}$ represent the vectors of the velocity distribution functions and design variables, respectively. The operator $\mathbf{M}$ performs one collision and one propagation step according to the LBE in Eq. (12). Here, the collision and propagation steps respectively represent collision operator $\mathbf{C}$ and propagation operator $\mathbf{P}$, and we define $\mathbf{M}=\mathbf{P}(\mathbf{C}): \mathbf{f}_{t} \rightarrow \mathbf{f}_{t+1}$, where index $t$ represents the time step of the LBE.

Objective function $\mathscr{F}=\mathscr{F}(\mathbf{f}, \mathbf{p})$ is now introduced in the optimization problem, and the design sensitivities that are the derivative of objective function $\mathscr{F}$ with respect to design variables $\mathbf{p}$ can be described as follows:

$$
\frac{\mathrm{d} \mathscr{F}}{\mathrm{d} \mathbf{p}}=\frac{\partial \mathscr{F}}{\partial \mathbf{p}}+\frac{\partial \mathscr{F} \mathrm{T}}{\partial \mathbf{f}} \frac{\mathrm{d} \mathbf{f}}{\mathrm{d} \mathbf{p}},
$$

where $\mathbf{f}$ is the velocity distribution function at the steady-state governed by the steady-state LBE in Eq. (40). Due to the large number of design variables, it would be computationally extravagant to compute the design sensitivities using the direct method by computing $\mathrm{d} / \mathrm{f} / \mathrm{d}$ for every design variable. In this case, the gradients of $\mathscr{F}$ can be efficiently computed using the adjoint variable method.

First, we differentiate the residual $\mathbf{R}$ in Eq. (40) with respect to the design variables $\mathbf{p}$,

$$
\frac{\partial \mathbf{R}}{\partial \mathbf{p}}+\frac{\partial \mathbf{R}}{\partial \mathbf{f}} \frac{\mathrm{d} \mathbf{f}}{\mathrm{d} \mathbf{p}}=0 .
$$

Thus, the gradients of $\mathbf{f}$ are given by,

$$
\frac{\mathrm{df}}{\mathrm{d} \mathbf{p}}=-\frac{\partial \mathbf{R}^{-1}}{\partial \mathbf{f}} \frac{\partial \mathbf{R}}{\partial \mathbf{p}} .
$$


Substituting Eq. (43) into (41), the sensitivity formulation based on the adjoint variable method is obtained as follows:

$$
\begin{aligned}
\mathbf{J}^{\mathrm{T}} \mathbf{a} & =\frac{\partial \mathscr{F}}{\partial \mathbf{f}}, \\
\frac{\mathrm{d} \mathscr{F}}{\mathrm{d} \mathbf{p}} & =\frac{\partial \mathscr{F}}{\partial \mathbf{p}}-\mathbf{a}^{\mathrm{T}} \frac{\mathrm{d} \mathbf{R}}{\mathrm{d} \mathbf{p}},
\end{aligned}
$$

where $\mathbf{J}=\partial \mathbf{R} / \partial \mathbf{f}$ is the Jacobian, and a represents the adjoint variables.

Based on the above sensitivity formulations, we can obtain the design sensitivities without computing $\mathrm{d} \mathscr{F} / \mathrm{d} p$ that incurs massive computational costs. However, in the above sensitivity formulations, the design sensitivities cannot be treated precisely, since the LBE, a so-called approximated equation, is used for the formulation of the optimization problems, and the derivation of their adjoint equation. Furthermore, even for the 2D9V model, the Jacobian $\mathbf{J}$ is a sparse asymmetric square matrix of size $(N \times 9)^{2}$ where $N$ is the number of lattice nodes. That is, time-consuming numerical operations are required to compute the Jacobian $\mathbf{J}$, and the advantages of the LBM, such as its algorithmic simplicity and computational efficiency, cannot be exploited in the adjoint equation.

\subsubsection{Proposed sensitivity formulation using the Boltzmann equation}

In this study, we construct a level set-based topology optimization method using the LBM, in which the optimization problems are formulated based on the original Boltzmann equation, in contrast to the formulation in a previous study [96] that is based on the LBE. The critical advantage obtained by using the Boltzmann equation is that the adjoint equation can be formulated as a continuous equation, in a manner similar to that used when formulating the Boltzmann equation. That is, both the adjoint equation and the LBE can be discretized in the same way, and the adjoint variable can be explicitly computed using a time evolution equation, rather than a matrix operation, during the optimization process.

First, we consider the variation of objective functional $\bar{J}$, which can be abstractly described as follows:

$$
\delta_{\phi} \bar{J}=\delta_{f} J[f]+\lambda \delta_{\phi} V[\phi]+\delta_{f} E[f, g, \phi]+\delta_{g} E[f, g, \phi]+\delta_{\phi} E[f, g, \phi],
$$

where the equilibrium equation $E$ is defined as $E=G+I+B_{n}$. In the above equation, the variation of $E$ with respect to the test function $g$ is equal to the original equilibrium equation, and can be eliminated since $E=0$. Arranging the above equation in order to eliminate the variation terms $\delta_{f} J[f]$ and $\delta_{f} E[f, g, \phi]$, we have the following:

$$
\delta_{f} J[f]+\delta_{f} E[f, g, \phi]=0,
$$

where $g$ that satisfies the above formulation is called the adjoint variable, and Eq. (47) is the adjoint equation. The above adjoint equation is then used to derive the following adjoint equations, following procedures detailed in Appendix B, as below:

$$
\begin{array}{cl}
-\frac{\partial g}{\partial t}-\xi \cdot \nabla g=-\frac{1}{\tau_{\mathrm{B}}}\left(g-g^{\mathrm{eq}}\right) & \text { in } I \times D \times \Xi, \\
\left.g\right|_{t=t_{1}}=-A^{\prime} & \text { in } D \times \Xi, \\
\left.g\right|_{t=t_{1}}=g_{\text {in }}^{\text {eq }}+a^{\prime} & \text { in } \Gamma_{\text {in }} \times \Xi, \\
\left.g\right|_{t=t_{1}}=g_{\text {out }}^{\text {eq }}+a^{\prime} & \text { in } \Gamma_{\text {out }} \times \Xi, \\
g(\boldsymbol{\xi})=g(-\boldsymbol{\xi}) & \text { in } I \times \Gamma_{\mathrm{w}} \times \Xi_{n \cdot \boldsymbol{\xi}}>0, \\
g=g_{\text {in }}^{\text {eq }} & \text { in } I \times \Gamma_{\text {in }} \times \Xi, \\
g=g_{\text {out }}^{\text {eq }} & \text { in } I \times \Gamma_{\text {out }} \times \Xi,
\end{array}
$$


where

$$
\begin{aligned}
g^{\mathrm{eq}} & =\int_{\Xi} g(\hat{\boldsymbol{\xi}}) \frac{T+\left(\chi_{\phi} \boldsymbol{u}-\boldsymbol{\xi}\right) \cdot\left(\chi_{\phi} \boldsymbol{u}-\hat{\boldsymbol{\xi}}\right)}{\rho T} \hat{f}^{\mathrm{eq}}(\hat{\boldsymbol{\xi}}) \mathrm{d} \hat{\boldsymbol{\xi}}, \\
g_{\text {in }}^{\mathrm{eq}} & =\int_{\Xi} g(\hat{\boldsymbol{\xi}}) \frac{1}{\rho} f^{\mathrm{eq}}(\hat{\boldsymbol{\xi}}) \mathrm{d} \hat{\boldsymbol{\xi}}, \\
g_{\text {out }}^{\mathrm{eq}} & =\int_{\Xi} g(\hat{\boldsymbol{\xi}}) \frac{\left(\chi_{\phi} \boldsymbol{u}-\boldsymbol{\xi}\right) \cdot\left(\chi_{\phi} \boldsymbol{u}-\hat{\boldsymbol{\xi}}\right)}{\rho_{\text {out }} T} f^{\mathrm{eq}}(\hat{\boldsymbol{\xi}}) \mathrm{d} \hat{\boldsymbol{\xi}}, \\
A^{\prime} & =\frac{1}{\rho}\left\{\frac{\partial A}{\partial \rho} \rho+\frac{\partial A}{\partial \boldsymbol{u}} \cdot(\boldsymbol{\xi}-\boldsymbol{u})\right\}, \\
a^{\prime} & =\frac{1}{\rho}\left\{\frac{\partial a}{\partial \rho} \rho+\frac{\partial a}{\partial \boldsymbol{u}} \cdot(\boldsymbol{\xi}-\boldsymbol{u})\right\} .
\end{aligned}
$$

Although the basic characteristics of the above adjoint formulation were introduced in Krause et al.'s approach [113], the inlet and outlet boundary conditions were not treated. To deal with these condition in our formulation, it can be used to formulate various optimization problems for the design of fluid channels. In addition, we note that the objective functional under a steady-state condition contributes to the initial condition of the adjoint equation in Eqs. (49), (50) and (51).

Since the level set function $\phi$ only depends on the equilibrium distribution function $f^{\mathrm{eq}}$, the design sensitivity of Eq. (46) is obtained as the following equation, using the adjoint variable $g$,

$$
\begin{aligned}
\delta_{\phi} \bar{J} & =\delta_{\phi} E[f, g, \phi]+\lambda \delta_{\phi} V[\phi] \\
& =\int_{T} \int_{D} \int_{\Xi} \frac{1}{\tau_{\mathrm{B}}} \frac{\partial \hat{f}^{\mathrm{eq}}}{\partial \phi} g \phi \mathrm{d} \boldsymbol{\xi} \mathrm{d} \Omega \mathrm{d} t+\lambda \int_{D} \delta \phi \mathrm{d} \Omega \\
& =\int_{D}\left\{\int_{T} \int_{\Xi} \frac{\left(\boldsymbol{\xi}-\chi_{\phi} \boldsymbol{u}\right) \cdot \boldsymbol{u}}{\tau_{\mathrm{B}} T} \hat{f}^{\mathrm{eq}} g \mathrm{~d} \boldsymbol{\xi} \mathrm{d} t+\lambda\right\} \delta \phi \mathrm{d} \Omega .
\end{aligned}
$$

We note that the proposed sensitivity formulations yield strictly correct sensitivities that are error free.

\section{Numerical implementation}

In this section, we first describe optimization algorithm used in this research. Then, we discuss a method for judging the steady-state flow condition, and the techniques to discretize the adjoint equation and the design sensitivities.

\subsection{Optimization algorithm}

The optimization algorithm of the proposed method is as follows:

(1) The initial level set function is set in the fixed design domain $D$

(2) The LBE is calculated until a steady-state condition is obtained.

(3) If the criteria of objective functional and volume constraint are satisfied, an optimal configuration is obtained and the optimization is finished, otherwise, the adjoint equation is calculated.

(4) The design sensitivities are calculated using the current velocity values, density values, and adjoint variables. The level set function is then updated based on Eq. (71), after which the optimization procedure returns to the second step of the iterative loop.

The updating of the level set function via the FEM employs the methodology used in previous research [59]. These procedures are iterated until the following criterion for the value of objective functional is met:

$$
\left|\frac{J_{\varsigma}-J_{\zeta-\Delta \varsigma}}{J_{\varsigma}}\right|<\varepsilon_{\mathrm{OPT}}
$$


where subscript $\varsigma$ represents the number of iterations carried out during the optimization process and $\Delta \varsigma$ represents a single iteration. We set the value of this criterion so that $\varepsilon_{\mathrm{OPT}}=1.0 \times 10^{-4}$. Concerning the calculation of the Lagrange multiplier $\lambda$ at each optimization iteration, when the volume constraint in Eq. (24) is active, the following equation is satisfied:

$$
\delta_{\phi} G=0 .
$$

Substituting Eq. (36) into (62), the Lagrange multiplier $\lambda$ is obtained as follows:

$$
\lambda=-\frac{\int_{D}\left(J^{\prime}+\tau \nabla^{2} \phi\right) \mathrm{d} \Omega}{\int_{D} \mathrm{~d} \Omega} .
$$

Here, the Lagrange multiplier $\lambda$ is updated using the following equation, to improve the convergence of the optimization calculation, in which the volume constraint $G$ is imposed:

$$
\bar{\lambda}=\lambda \exp ^{G} .
$$

We note that since the exponential function $\exp ^{G}$ can be expanded, using a Taylor expansion around $G=0$, as $\exp ^{G}=$ $1+G+1 / 2 G^{2}+\cdots$, the above equation for $\bar{\lambda}$ is based on the augmented Lagrangian method [114], in which the $\lambda$ is updated using $\bar{\lambda}=\lambda+c G$, where $c \in \mathbb{R}_{\geqslant 0}$ is a penalty parameter. When the volume constraint $G$ is inactive, the Lagrange multiplier automatically assumes a value of $\lambda \approx 0$ due to the use of the exponential function in Eq. (64).

\subsection{Judgment of steady-state condition}

Since the LBM is an explicit time evolution scheme for computing transient flows, in order to evaluate the objective functional $J$ in a steady-state condition, we employ the following criterion,

$$
\left\|\frac{\boldsymbol{u}_{\varsigma}-\boldsymbol{u}_{\varsigma-\Delta \zeta}}{\boldsymbol{u}_{\varsigma}}\right\|_{L^{2}(D)}<\varepsilon_{\mathrm{LBM}},
$$

We use $\varepsilon_{\mathrm{LBM}}=1.0 \times 10^{-4}$ as a criterion to judge the steady-state condition in this research.

\subsection{Discretization of the adjoint equation and design sensitivities}

Due to the similar configuration of the Boltzmann equation (48) and the adjoint equation (17), the discrete adjoint equation can be formulated as follows, as was done to obtain the discrete Boltzmann equation (5), for the adjoint variable $g_{i}(\boldsymbol{x}, t)=g\left(\boldsymbol{x}, t, \boldsymbol{c}_{i}\right)$.

$$
-\mathrm{Sh} \frac{\partial g_{i}}{\partial t}-\boldsymbol{c}_{i} \cdot \nabla g_{i}=-\frac{1}{\tau_{\mathrm{B}}}\left(g_{i}-g_{i}^{\mathrm{eq}}\right) \quad \text { in } \quad I \times \Omega \times \Xi^{h} .
$$

Applying a Gauss-Hermite quadrature to $g^{\mathrm{eq}}$ in Eq. (66), $g_{i}^{\mathrm{eq}}$ is obtained as

$$
g_{i}^{\mathrm{eq}}=\sum_{j=1}^{q} g_{i}\left(\boldsymbol{c}_{j}\right) \frac{1+\left(\chi_{\phi} \boldsymbol{u}-\boldsymbol{c}_{i}\right) \cdot\left(\chi_{\phi} \boldsymbol{u}-\boldsymbol{c}_{j}\right)}{\rho} \hat{f}_{j}^{\mathrm{eq}},
$$

in which we assume that the temperature is $T=1$ that means the dimensional temperature $\bar{T}$, which is assumed the isothermal condition, is equal to the reference temperature $T_{0}$.

Now, applying the discretization strategy used in Eq. (11), the above equation (66) is discretized as follows:

$$
\begin{aligned}
& \frac{g_{i}(x, y, z, t-\Delta t)-g_{i}(x, y, z, t)}{\Delta t} \\
& \quad+\frac{c_{i x}}{\operatorname{Sh}} \frac{g_{i}\left(x-c_{i x} \Delta x, y-c_{i y} \Delta x, z-c_{i z} \Delta x, t-\Delta t\right)-g_{i}\left(x, y-c_{i y} \Delta x, z-c_{i z} \Delta x, t-\Delta t\right)}{c_{i x} \Delta x} \\
& \quad+\frac{c_{i y}}{\operatorname{Sh}} \frac{g_{i}\left(x, y-c_{i y} \Delta x, z-c_{i z} \Delta x, t-\Delta t\right)-g_{i}\left(x, y, z-c_{i z} \Delta x, t-\Delta t\right)}{c_{i y} \Delta x} \\
& \quad+\frac{c_{i z}}{\operatorname{Sh}} \frac{g_{i}\left(x, y, z-c_{i z} \Delta x, t-\Delta t\right)-g_{i}(x, y, z, t-\Delta t)}{c_{i z} \Delta x} \\
& =-\frac{1}{\tau_{\mathrm{B}} \operatorname{Sh}}\left\{g_{i}(x, y, z, t)-g_{i}^{\mathrm{eq}}(x, y, z, t)\right\} .
\end{aligned}
$$


Since the time step is expressed as $\Delta t=\operatorname{Sh} \Delta x$ in the LBE, we now obtain the following discretized adjoint equation:

$$
g_{i}\left(\boldsymbol{x}-\boldsymbol{c}_{i} \Delta x, t-\Delta t\right)=g_{i}(\boldsymbol{x}, t)-\frac{1}{\tau_{\mathrm{B}}}\left\{g_{i}(\boldsymbol{x}, t)-g_{i}^{\mathrm{eq}}(\boldsymbol{x}, t)\right\} \quad \text { in } I^{h} \times D^{h} \times \Xi^{h} .
$$

Similarly, using the particle velocities $\boldsymbol{c}_{i}$ and the discretized variables $\hat{f}_{i}^{\mathrm{eq}}$ and $g_{i}$, the design sensitivities in Eq. (60) can be represented as follows:

$$
\delta_{\phi} \bar{J}=\int_{D}\left\{\frac{1}{\tau_{\mathrm{B}}} \sum_{i=1}^{q} \hat{f}_{i}^{\mathrm{eq}} g_{i}\left\{\boldsymbol{c}_{i}-\chi_{\phi} \boldsymbol{u}\right\} \cdot \boldsymbol{u}+\lambda\right\} \delta \phi \mathrm{d} \Omega .
$$

Therefore, the time evolutionary equation (36) of the optimization problems using the Boltzmann equation is

$$
\frac{\partial \phi}{\partial \varsigma}=-K\left\{\frac{1}{\tau_{\mathrm{B}}} \sum_{i=1}^{q} \hat{f}_{i}^{\mathrm{eq}} g_{i}\left\{\boldsymbol{c}_{i}-\chi_{\phi} \boldsymbol{u}\right\} \cdot \boldsymbol{u}+\lambda-\tau \nabla^{2} \phi\right\} .
$$

We note that the discretized adjoint equation, (69), allows us to avoid having to deal with matrix operations to obtain the design sensitivities during the optimization process.

\section{Numerical examples}

In this section, two- and three-dimensional numerical examples are provided. All numerical examples use the same parameters for the optimization: $\tau=5.0 \times 10^{-3}, K=1, \Delta \varsigma=0.25$, and $\phi^{0}=1$, which sets the initial configuration so that the fixed design domain $D$ is filled with fluid. In addition, since all numerical examples are treated as internal channel flow problems, the boundaries of $D$ are set to a non-slip condition where $\boldsymbol{u}=\mathbf{0}$.

\subsection{Comparison of LBM and FEM results}

First, we confirm the applicability of our methodology by comparing an obtained result with that obtained using a previous FEM-based approach [115]. In this case, we use the following objective functional,

$$
\left.J_{1}\right|_{t=t_{1}}=\int_{D} \frac{1}{2 \operatorname{Re}}\left\{\nabla \boldsymbol{u}+(\nabla \boldsymbol{u})^{\mathrm{T}}\right\}:\left\{\nabla \boldsymbol{u}+(\nabla \boldsymbol{u})^{\mathrm{T}}\right\} \mathrm{d} \Omega,
$$

which represents the dissipation energy of the flow, with Re representing the Reynolds number in the flow field. The terms in the adjoint equations (58) and (59) are derived as follows:

$$
\begin{aligned}
& A^{\prime}=\frac{1}{\operatorname{Re} \rho}\left\{\nabla \boldsymbol{u}+(\nabla \boldsymbol{u})^{\mathrm{T}}\right\}:\left\{\nabla \boldsymbol{u}+(\nabla \boldsymbol{u})^{\mathrm{T}}\right\}, \\
& a^{\prime}=0 .
\end{aligned}
$$

The design settings for the two-dimensional channel flow problem are shown in Fig. 4(a). The fluid velocity at the two left inlets is defined as $\boldsymbol{u}_{\mathrm{I}}=\left(U_{0}, 0\right)^{\mathrm{T}}$ using the inlet velocity $U_{0}=1.2 \times 10^{-2}$, and the pressure at the two left outlets is set as $p_{0}=0.33$. The volume constraint $V_{\max }=(1 / 3) V_{0}$ (where $V_{0}$ is the volume of the fixed design domain $D$ ), and the relaxation time $\tau_{\mathrm{B}}=0.8$, which is a physically appropriate value used to represent the fluid properties as a continuum. The kinematic viscosity of the fluid is then obtained as $v=\left(\tau_{\mathrm{B}}-1 / 2\right) \Delta x / 3=2.0 \times 10^{-3}$. Hence, the Reynolds number is represented as $\operatorname{Re}=U_{0} L_{0} / v=1$, where we define the characteristic length $L_{0}$ as the width of the inlet.

Figure 5 shows the velocity field and the adjoint velocity field that is defined as $\tilde{\boldsymbol{u}}=\sum_{i=1}^{q} \boldsymbol{c}_{i} g$ in the first step of the iterated optimization procedure for the flow channel design problem shown in Fig. 4(a). We note that the direction of the adjoint velocity $\tilde{\boldsymbol{u}}$ is opposite that of the velocity $\boldsymbol{u}$. This relationship mirrors that between the directions of particle velocities $\boldsymbol{c}_{i}$ in Eq. (66), which are opposite those of the particle velocities in the Boltzmann equation. In Navier-Stokes flow problems, Katamine et al. [4] and Zhou and Li [52] have shown that in flow friction minimization problems, the adjoint variable and the fluid velocity have the same characteristics, namely that their vectors are 


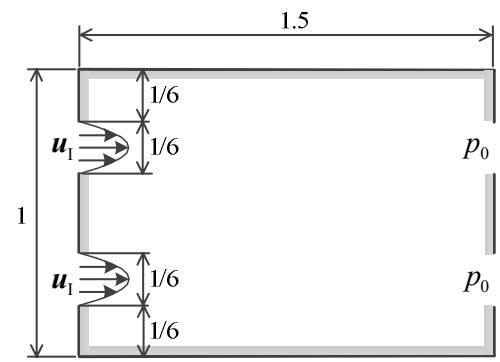

(a) Design settings

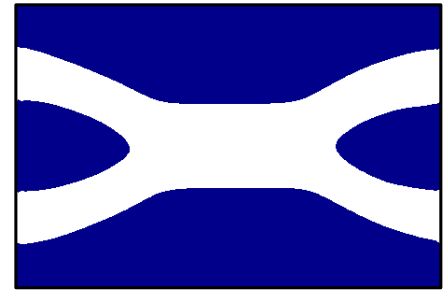

(b) LBM

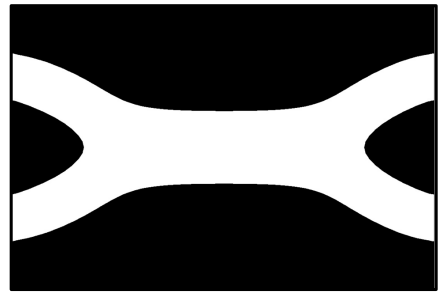

(c) FEM

Figure 4: Comparison of LBM and FEM results

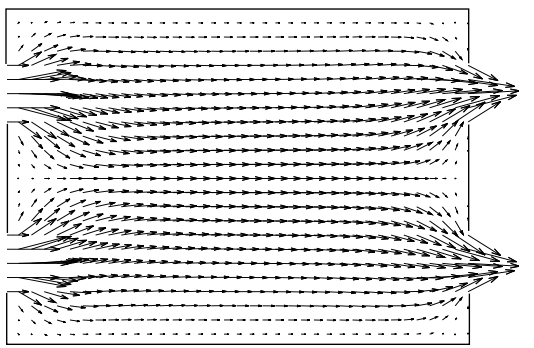

(a) Velocity field

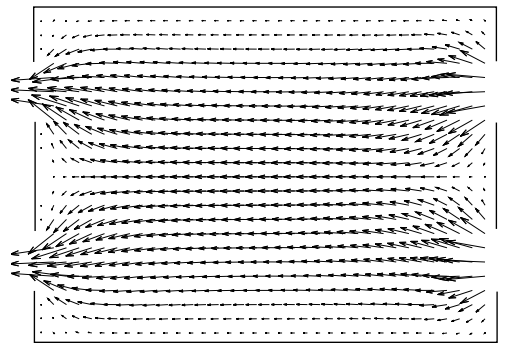

(b) Adjoint velocity field

Figure 5: Velocity and adjoint velocity fields in the first step of optimization iteration of flow channel design problem in Fig. 4(a). The definition of the adjoint velocity is $\tilde{\boldsymbol{u}}=\sum_{i=1}^{q} \boldsymbol{c}_{i} g$.

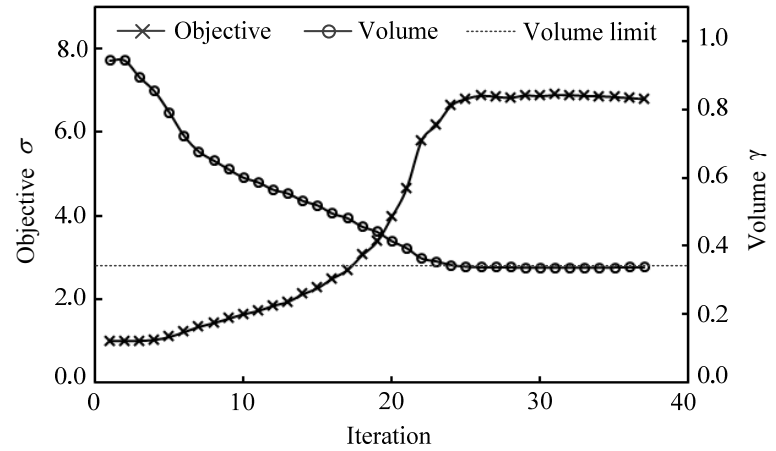

Figure 6: Convergence history of objective functional $J_{1}$ values and volume fraction. $\sigma=J_{1} / J_{1}^{0}$ represents the ratio of the values of current objective functionals $J_{1}$ to the value of the initial objective functional $J_{1}^{0} \cdot \gamma=V / V_{0}$ represents the volume ratio of the current volume of the fluid domain $V$ to the initial volume $V_{0}$.

opposite in direction. Thus, $\tilde{\boldsymbol{u}}$ can be considered as corresponding to the adjoint variable in optimization problems that incorporate the Navier-Stokes equations.

Figures 4(b) and (c) show the optimal configurations based on the LBM (b) and FEM (c) approaches for the flow channel problem. The general similarity of the configurations confirms that the proposed LBM can obtain appropriate results. In the LBM-based method, the expanded Maxwell distribution in Eq. (15) is used to express the solid boundaries. On the other hand, the FEM-based method uses an artificial force based on Darcy's law [116]. Thus, 


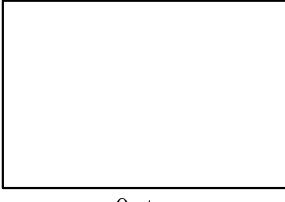

0 step

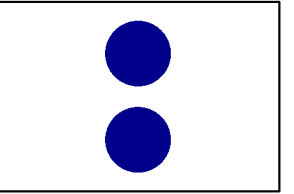

0 step

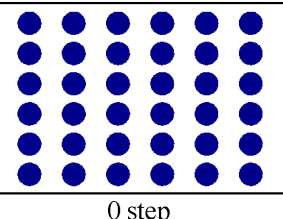

0 step

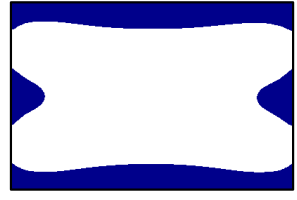

5 step

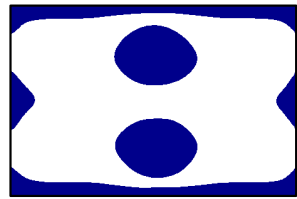

5 step

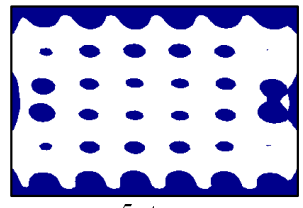

5 step

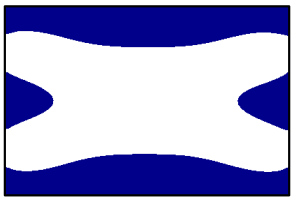

10 step

Case 1

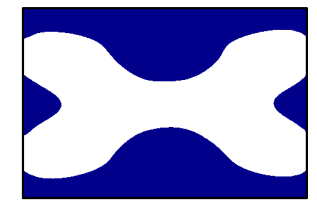

10 step

Case 2

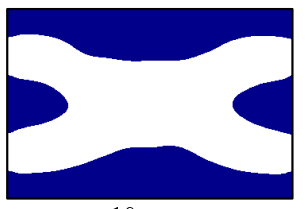

10 step

Case 3

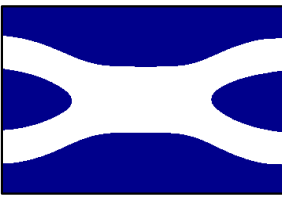

20 step

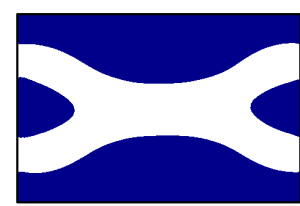

20 step

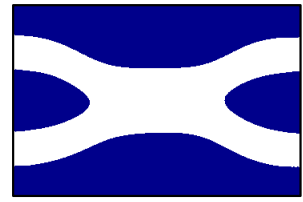

20 step

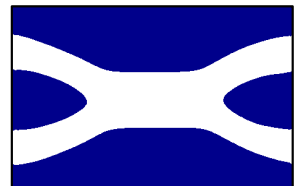

Optimal configuration

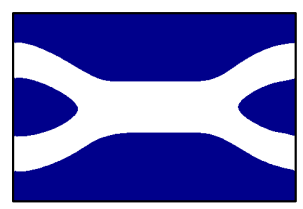

Optimal configuration

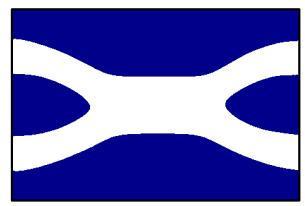

Optimal configuration

Figure 7: Optimization histories and optimal configurations for three initial configurations

we note that the difference between the LBM and FEM optimal configurations may be a consequence of the different definitions used in the treatment of the solid domain.

Figure 6 shows the convergence history of the value of the objective functional $J_{1}$ and the volume fraction. We can confirm that both are smoothly converged and that the optimal configuration is a valid solution.

\subsection{Dependency of optimal configurations on initial configurations}

Next, we examine the dependency of the optimal configurations with respect to the initial configurations, using the design model shown in Fig. 4(a). In conventional approaches based on the level set method, dependency on initial configurations is considered to be a fatal disadvantage from the standpoint of obtaining optimal configurations [25]. The main reason why this problem occurs is that the Hamilton-Jacobi equation is used for updating the level set function. Due to the characteristics of this equation, conventional approaches are essentially shape optimizations; that is, topological changes, such as the generation of a new hole (in the fluid or solid domains), are disallowed during the optimization process. On the other hand, since we use a reaction-diffusion equation (36) when updating the level set function, topological changes as well as changes in structural boundaries are allowed during the optimization process. Here, we treat three cases, each using a different initial configuration, using a grid size of $\Delta x=2.0 \times 10^{-2}$ and the same Reynolds number condition as that applied in the previous problem described in section 5.2.

Figure 7 shows the three different initial settings and configurations at selected iterations prior to obtaining optimal configurations. Although each initial configuration has a different topology, the obtained optimal configurations have the same topology and a similar configuration. The similarity of the optimal configurations shown in Fig. 7 indicates that dependency with respect to the initial configuration is low.

\subsection{Dependency of optimal configurations on grid size}

Next, we examine the dependency of the optimal configurations with respect to the grid size, using the design model shown in Fig. 8(a). Here, two cases are treated, using grid sizes of $\Delta x=2.0 \times 10^{-2}$ and $\Delta x=1.0 \times 10^{-2}$ under the same volume constraint condition, with $V_{\max }=(2 / 5) V_{0}$. 


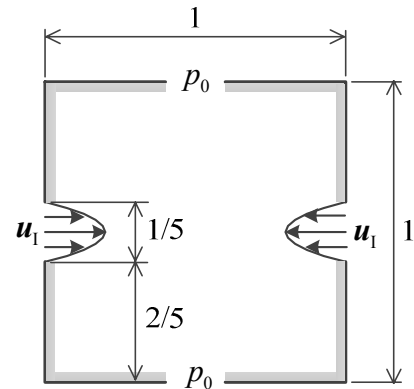

(a) Design settings

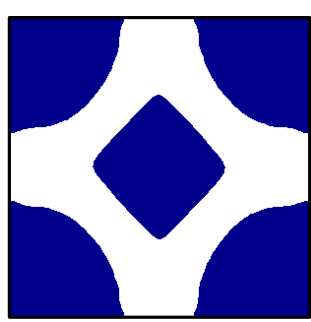

(b) $50 \times 50$ lattice

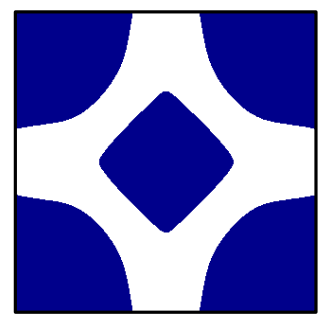

(c) $100 \times 100$ lattice

Figure 8: Dependency of optimal configurations on grid size

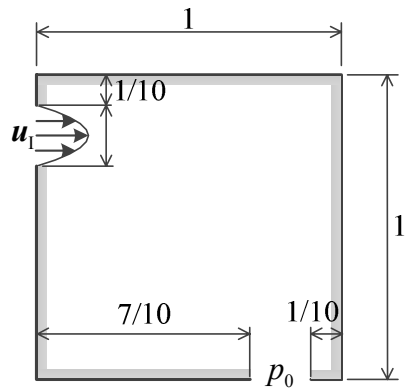

(a) Design settings

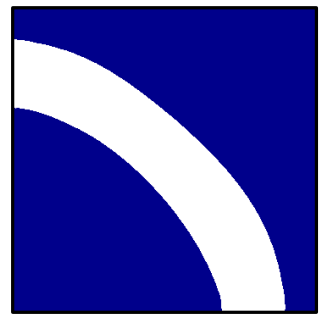

(b) Dissipation energy

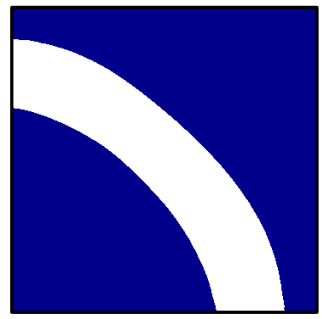

(c) Pressure drop

Figure 9: Design settings and optimal configurations for pipe bend problem

Here, the Reynolds number is set as $\mathrm{Re}=10$ in order to ensure that the Reynolds number condition is the same in both cases that use different grid sizes.

The similarity of the optimal configurations in Fig. 8(b) and (c) indicates that dependency with respect to grid size is low.

\subsection{Objective functional for pressure drop between inlet and outlet}

To address a second problem, minimization of the pressure drop between inlet and outlet, we use the following objective functional that expresses the pressure drop between inlet $\Gamma_{\text {in }}$ and outlet $\Gamma_{\text {out }}$ :

$$
\left.J_{2}\right|_{t=t_{1}}=\int_{\Gamma_{\text {in }}} p \mathrm{~d} \Gamma-\int_{\Gamma_{\text {out }}} p \mathrm{~d} \Gamma .
$$

Using $J_{2}$, this optimization problem is equivalent to a problem to minimize dissipation $J_{1}$, from a physical standpoint. However, we note that the objective functional $J_{2}$ can only be used in slow velocity regimes, where $|\boldsymbol{u}| \ll 1$, since the dynamic pressure $\rho|\boldsymbol{u}|^{2} / 2$ is neglected. The terms in the adjoint equations (58) and (59) are derived as follows:

$$
\begin{aligned}
& A^{\prime}=0, \\
& a^{\prime}=\left\{\begin{array}{lll}
\frac{1}{3} & \text { on } & \Gamma_{\text {in }}, \\
-\frac{1}{3} & \text { on } & \Gamma_{\text {out }} .
\end{array}\right.
\end{aligned}
$$

Figure 9 shows the design settings and optimal configurations for a pipe bend problem to examine the difference in the obtained results when the goal is to minimize the dissipation energy or the pressure drop, using objective 


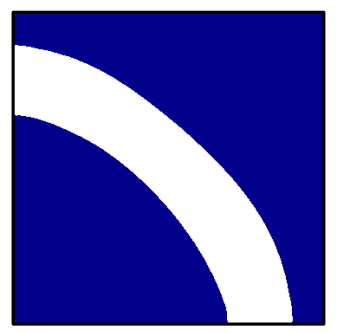

(a) $\operatorname{Re}=5$

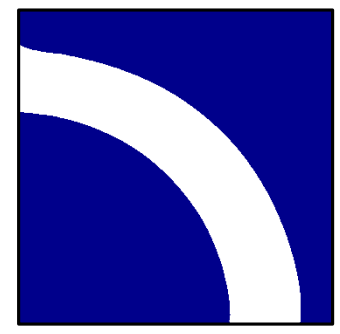

(b) $\operatorname{Re}=150$

Figure 10: Dependency of optimal configurations on Reynolds number in pipe bend problem

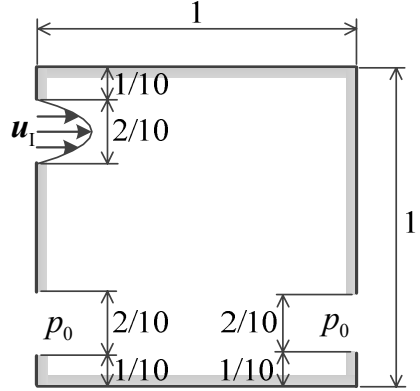

(a) Design settings

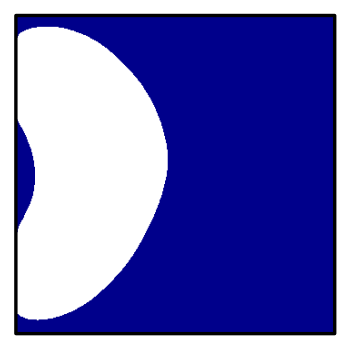

(b) $\mathrm{Re}=1$

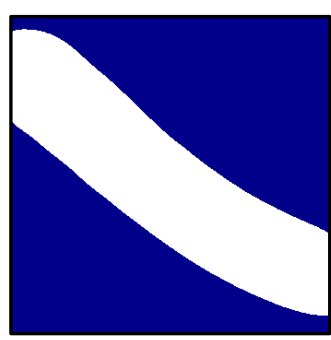

(c) $\operatorname{Re}=50$

Figure 11: Dependency of optimal configurations on Reynolds number in switching pipe problem

functionals $J_{1}$ and $J_{2}$, respectively. Identical flow properties and grid size $\Delta x=2.0 \times 10^{-2}$, with Reynolds number $\operatorname{Re}=5$, were used in both cases. The volume constraint in both cases was set to the same value, $V_{\max }=(1 / 4) V_{0}$. The close similarity of the optimal configurations in Fig. 9(b) and (c) indicates that the minimization problem for the flow friction in our topology optimization method can be formulated using the pressure drop that is formulated as a boundary integration, without using the derivative term.

\subsection{Dependency of optimal configurations on Reynolds number}

Here, we examine the dependency of the optimal configurations with respect to different Reynolds numbers, using the objective functional $J_{1}$ in Eq. (74).

Figure 10 shows the optimal configurations obtained for two different Reynolds numbers in the pipe bend problem: (a) $\operatorname{Re}=5$; and (b) $\operatorname{Re}=150$. The differences in the optimal configurations indicate that the value of the Reynolds number affects the channel's configuration; the radius of curvature is decreased as the Reynolds number is increased.

Next, we treat the pipe flow problem shown in Fig. 11, where (a), (b), and (c) are the design model, the optimal configuration under $\operatorname{Re}=1$, and the optimal configuration under $\operatorname{Re}=50$, respectively. In this problem, the volume constraint is set with $V_{\max }=(2 / 5) V_{0}$. In the low Reynolds number case, the optimal channel configuration is an arc shape connecting the inlet to the outlet of the left side boundary. On the other hand, in the high Reynolds number case, the optimal channel configuration is an essentially straight shape connecting the inlet with the outlet at the right side boundary.

\subsection{Three-dimensional channel flow problems}

Finally, the previous two-dimensional cases are extended to three-dimensional channel flow problems. Here, we consider two design problems, using objective functional $J_{2}$ in Eq. (75). 


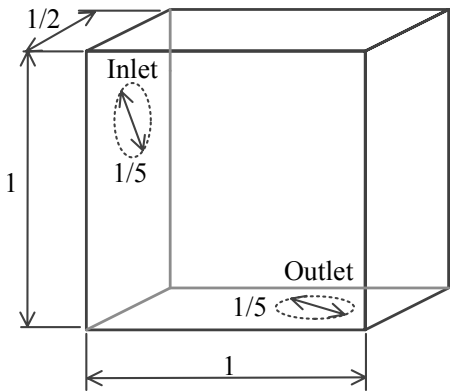

(a) Design settings
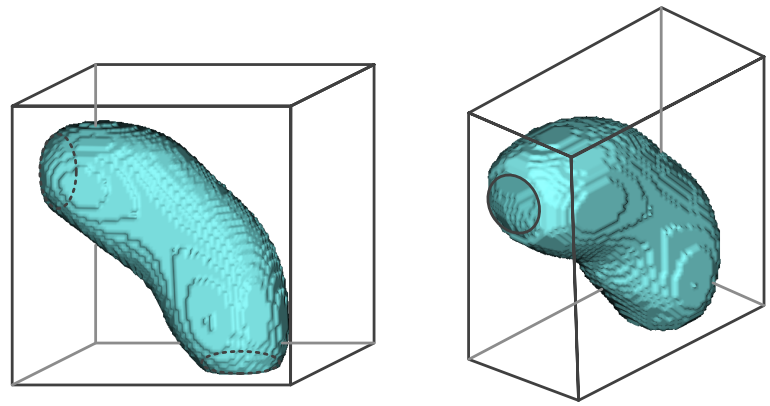

(b) Optimal configuration

Figure 12: Optimal configuration in three-dimensional pipe bend problem

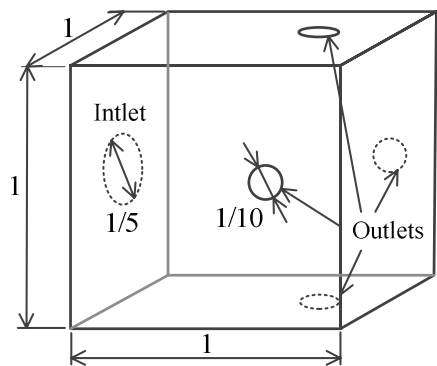

(a) Design settings
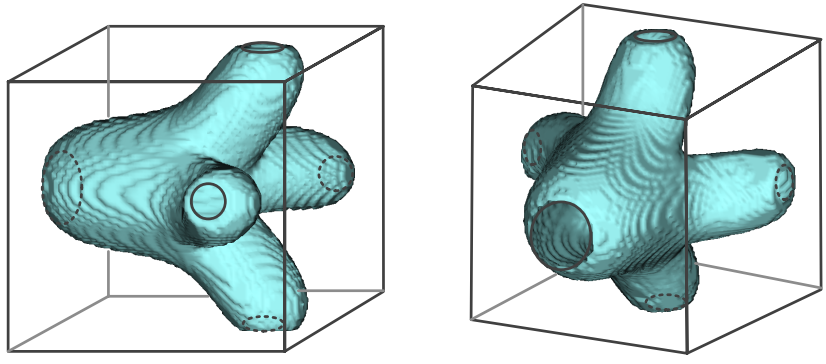

(b) Optimal configuration

Figure 13: Optimal configuration in three-dimensional multi-outlet problem

First, we consider the three-dimensional pipe bend problem shown in Fig. 12(a), in which the volume constraint $V_{\max }=(1 / 3) V_{0}$, and cubic grid size $\Delta x=1.25 \times 10^{-3}$. Since the reference length in this problem is the diameter of the inlet, the Reynolds number is set as $\mathrm{Re}=20$. Figure 12(b) shows the optimal configuration for the three-dimensional pipe bend problem.

In the second numerical example for a three-dimensional case, we consider a problem with a single inlet and four outlets, as shown in Fig. 13(a). In this problem, the volume constraint, cubic grid size, and Reynolds number are respectively set as $V_{\max }=(2 / 5) V_{0}, \Delta x=1.25 \times 10^{-3}$, and $\mathrm{Re}=20$. Figure 13(b) shows the optimal configuration for the three-dimensional multi-outlet problem.

These optimal configurations indicate that the proposed method can derive a valid optimal structure in a threedimensional case.

\section{Conclusion}

This paper presented a new level set-based topology optimization method for fluid dynamics problems using the LBM. The presented method was applied to flow friction minimization problems for incompressible viscous flow. We achieved the following:

(1) A topology optimization method was formulated, incorporating level set boundary expressions, where the sensitivity formulations, based on the original Boltzmann equation, enable the design sensitivities to be precisely 
derived without matrix operations, in contrast to previous research in which the use of the LBM required largescale matrix operations.

(2) Based on the formulation of the optimization problem, an optimization algorithm was constructed. Due to the similar configuration of the Boltzmann and adjoint equations, the adjoint equation was discretized as an explicit time evolutional equation, using the discretization strategy applied to the LBE. The time evolution equation for updating the level set function was solved using the FEM.

(3) Several numerical examples for both two- and three-dimensional problems were provided to confirm the validity and utility of the presented method, for dissipation energy minimization and pressure drop minimization problems. Based on the obtained results, we confirmed that the presented method obtains smooth and clear optimal configurations that show minimal dependency upon the initial configurations and mesh size. We also demonstrated that the presented method obtains an optimal configuration similar to that when using an FEMbased method. In addition, we confirmed that optimal configurations obtained by the presented method show dependency with respect to Reynolds number settings.

\section{Acknowledgments}

This work was supported in part by a Grant for Environmental Research Projects from The Sumitomo Foundation, and JSPS KAKENHI Grant Number 24760119.

\section{Appendix A. Definition of non-dimensional variables}

We use the following non-dimensional variables defined by a characteristic length $L$, a characteristic particle speed $c$, a characteristic time $\tilde{t}=L / U$ where $U$ is a characteristic flow speed, a reference order parameter $f_{0}$, a reference density $\rho_{0}$, and a reference temperature $T_{0}$, as follows:

$$
\boldsymbol{x}=\frac{\overline{\boldsymbol{x}}}{L}, \quad \boldsymbol{\xi}=\frac{\overline{\boldsymbol{\xi}}}{c}, \quad t=\frac{\bar{t}}{\bar{t}}, \quad f=\frac{\bar{f}}{f_{0}}, \quad \rho=\frac{\bar{\rho}}{\rho_{0}}, \quad T=\frac{\bar{T}}{T_{0}}, \quad \boldsymbol{u}=\frac{\overline{\boldsymbol{u}}}{c}, \quad p=\frac{\bar{p}}{\rho_{0} c^{2}}, \quad v=\frac{\overline{\boldsymbol{v}}}{c L},
$$

where, $\overline{\boldsymbol{x}}, \overline{\boldsymbol{\xi}}, \bar{t}, \bar{f}, \bar{\rho}, \bar{T}, \overline{\boldsymbol{u}}, \bar{p}, \bar{v}$ represent dimensional quantities for each variable. In this study, the characteristic particle speed is set to $c=\sqrt{2 R T_{0}}$, where $R$ represents the specific gas constant and $c$ is a parameter of the same order as that of the speed of sound in the specific gas at temperature $T_{0}$. Based on the dimensional Maxwell distribution with $\boldsymbol{u}=\mathbf{0}$, the reference order parameter $f_{0}$ is defined as follows:

$$
f_{0}=\frac{\rho_{0}}{\left(2 \pi R T_{0}\right)^{d / 2}} \exp \left(-\frac{c^{2}}{2 R T_{0}}\right)
$$

\section{Appendix B. Derivation of the adjoint equation}

Here, we discuss the details concerning the derivation of the adjoint equation in Eq. (48), and the initial and boundary conditions expressed in Eqs. (49), (52), (53), and (54). We consider the specific formulation of each term in the left side of Eq. (47).

First, we derive the specific formulation of $\delta_{f} J[f]$, which is represented as the following variation of the objective functional:

$$
\delta_{f} J[f]=\lim _{\varepsilon \rightarrow 0} \frac{J[f+\varepsilon \eta]-J[f]}{\varepsilon} \text { for } \forall \eta \in \mathscr{U},
$$

where $\mathscr{U}$ represents the function space of arbitrary function $\eta$, and Eq. (B.1) is based on the Gâtaux derivative. To ensure a precise derivation, we define function space $\mathscr{U}$ as follows:

$$
\mathscr{U}=\left\{\eta \mid \eta \in H^{1}(I \times D \times \Xi) \text { with } \eta(\xi)=\eta(-\boldsymbol{\xi}) \text { on } \Gamma_{\mathrm{w}}\right\}
$$


In addition, the objective functional is divided, with $J:=J_{D}+J_{\Gamma}$. These two functionals are then defined as follows: $J_{D}=\int_{D} A(\rho, \boldsymbol{u}) \mathrm{d} \Omega$, and $J_{\Gamma}=\int_{\Gamma_{\mathrm{in}} \cup \Gamma_{\text {out }}} a(\rho, \boldsymbol{u}) \mathrm{d} \Gamma$. Based on Eq. (B.1), $J_{D}[f+\varepsilon \eta]$ is derived as follows:

$$
J_{D}[f+\varepsilon \eta]=J_{D}[A(\rho[f+\varepsilon \eta], \boldsymbol{u}[f+\varepsilon \eta])]=J_{D}[A\{\rho[f+\varepsilon \eta], \boldsymbol{u}(\rho[f+\varepsilon \eta], \boldsymbol{v}[f+\varepsilon \eta])\}],
$$

and we define $\boldsymbol{u}$ as

$$
\begin{aligned}
\boldsymbol{u} & =\frac{\boldsymbol{v}}{\rho}, \\
\boldsymbol{v} & =\int_{\Xi} \boldsymbol{\xi} f \mathrm{~d} \boldsymbol{\xi} .
\end{aligned}
$$

Since $\rho$ and $v$ are linear functionals, the integrand $A$ in Eq. (B.3) can be rewritten as

$$
A\{\rho[f+\varepsilon \eta], \boldsymbol{u}(\rho[f+\varepsilon \eta], \boldsymbol{v}[f+\varepsilon \eta])\}=A\{\rho[f]+\varepsilon \rho[\eta], \boldsymbol{u}(\rho[f]+\varepsilon \rho[\eta], \boldsymbol{v}[f]+\varepsilon \boldsymbol{v}[\eta])\},
$$

where $\boldsymbol{u}(\rho[f]+\varepsilon \rho[\eta], \boldsymbol{v}[f]+\boldsymbol{\varepsilon v}[\eta])$ is expanded as follows, using the Taylor expansion:

$$
\boldsymbol{u}(\rho[f]+\varepsilon \rho[\eta], \boldsymbol{v}[f]+\boldsymbol{\varepsilon} \boldsymbol{v}[\eta])=\boldsymbol{u}(\rho[f], \boldsymbol{v}[f])+\varepsilon\left\{\frac{\partial \boldsymbol{u}}{\partial \rho} \rho[\eta]+\frac{\partial \boldsymbol{u}}{\partial \boldsymbol{v}} \boldsymbol{v}[\eta]\right\}+o(\varepsilon) .
$$

Furthermore, using Eq. (B.7), Eq. (B.6) can also be expanded as follows, using the Taylor expansion:

$$
\begin{aligned}
A\{\rho[f]+ & \varepsilon \rho[\eta], \boldsymbol{u}(\rho[f]+\varepsilon \rho[\eta], \boldsymbol{v}[f]+\varepsilon \boldsymbol{v}[\eta])\} \\
& =A\{\rho[f], \boldsymbol{u}(\rho[f], \boldsymbol{v}[f])\}+\varepsilon\left\{\frac{\partial A}{\partial \rho} \rho[\eta]+\frac{\partial A}{\partial \boldsymbol{v}} \cdot\left(\frac{\partial \boldsymbol{u}}{\partial \rho} \rho[\eta]+\frac{\partial \boldsymbol{u}}{\partial \boldsymbol{v}} \boldsymbol{v}[\eta]\right)\right\}+o(\varepsilon),
\end{aligned}
$$

where

$$
\begin{aligned}
& \frac{\partial \boldsymbol{u}}{\partial \rho}=-\frac{\boldsymbol{v}}{\rho^{2}} \\
& \frac{\partial \boldsymbol{u}}{\partial \boldsymbol{v}}=\frac{1}{\rho} .
\end{aligned}
$$

Hence, based on Eq. (B.1), the variation $\delta_{f} J_{D}$ is obtained as follows:

$$
\begin{aligned}
\delta_{f} J_{D} & =\lim _{\varepsilon \rightarrow 0} \frac{J_{D}[f+\varepsilon \eta]-J_{D}[f]}{\varepsilon} \\
& =\int_{D}\left\{\frac{\partial A}{\partial \rho} \rho[\eta]+\frac{\partial A}{\partial \boldsymbol{v}} \cdot\left(\frac{\partial \boldsymbol{u}(\rho[f], \boldsymbol{v}[f])}{\partial \rho} \rho[\eta]+\frac{\partial \boldsymbol{u}(\rho[f], \boldsymbol{v}[f])}{\partial \boldsymbol{v}} \boldsymbol{v}[\eta]\right)\right\} \mathrm{d} \Omega \\
& =\int_{D}\left\{\frac{\partial A}{\partial \rho} \rho[\eta]+\frac{\partial A}{\partial \boldsymbol{v}} \cdot\left(-\frac{\boldsymbol{v}[f]}{(\rho[f])^{2}} \rho[\eta]+\frac{1}{\rho[f]} \boldsymbol{v}[\eta]\right)\right\} \mathrm{d} \Omega \\
& =\int_{D}\left\{\frac{\partial A}{\partial \rho} \rho[\eta]+\frac{\partial A}{\partial \boldsymbol{v}} \cdot\left(-\frac{\boldsymbol{u}(\rho[f], \boldsymbol{v}[f])}{\rho[f]} \rho[\eta]+\frac{\boldsymbol{u}(\rho[\eta], \boldsymbol{v}[\eta])}{\rho[f]} \rho[\eta]\right)\right\} \mathrm{d} \Omega \\
& =\int_{D} \frac{1}{\rho[f]}\left\{\frac{\partial A}{\partial \rho} \rho[f]+\frac{\partial A}{\partial \boldsymbol{v}} \cdot\{\boldsymbol{u}(\rho[\eta], \boldsymbol{v}[\eta])-\boldsymbol{u}(\rho[f], \boldsymbol{v}[f])\}\right\} \rho[\eta] \mathrm{d} \Omega \\
& =\int_{D} \int_{\Xi} \frac{1}{\rho[f]}\left\{\frac{\partial A}{\partial \rho} \rho[f]+\frac{\partial A}{\partial \boldsymbol{v}} \cdot\{\boldsymbol{\xi}-\boldsymbol{u}(\rho[f], \boldsymbol{v}[f])\}\right\} \eta \mathrm{d} \boldsymbol{\xi} \mathrm{d} \Omega,
\end{aligned}
$$

with the following functional used with respect to the arbitrary function $\eta$ :

$$
\begin{gathered}
\rho[\eta]=\int_{\Xi} \eta \mathrm{d} \boldsymbol{\xi}, \\
\boldsymbol{u}(\rho[\eta], \boldsymbol{v}[\eta])=\frac{1}{\rho[\eta]} \int_{\Xi} \boldsymbol{\xi} \eta \mathrm{d} \boldsymbol{\xi} .
\end{gathered}
$$


Similarly, $\delta_{f} J_{\Gamma}$ can be derived as follows:

$$
\begin{aligned}
\delta_{f} J_{\Gamma} & =\lim _{\varepsilon \rightarrow 0} \frac{J_{\Gamma}[f+\varepsilon \eta]-J_{\Gamma}[f]}{\varepsilon} \\
& =\int_{\Gamma_{\text {in }} \cup \Gamma_{\text {out }}} \int_{\Xi} \frac{1}{\rho[f]}\left\{\frac{\partial a}{\partial \rho} \rho[f]+\frac{\partial a}{\partial \boldsymbol{v}} \cdot\{\boldsymbol{\xi}-\boldsymbol{u}(\rho[f], \boldsymbol{v}[f])\}\right\} \eta \mathrm{d} \boldsymbol{\xi} \mathrm{d} \Gamma .
\end{aligned}
$$

Consequently, we obtain the following variation, $\delta_{f} J=\delta_{f} J_{D}+\delta_{f} J_{\Gamma}$, as

$$
\begin{aligned}
\delta_{f} J= & \int_{D} \int_{\Xi} \frac{1}{\rho[f]}\left\{\frac{\partial A}{\partial \rho} \rho[f]+\frac{\partial A}{\partial \boldsymbol{v}} \cdot\{\boldsymbol{\xi}-\boldsymbol{u}(\rho[f], \boldsymbol{v}[f])\}\right\} \eta \mathrm{d} \boldsymbol{\xi} \mathrm{d} \Omega \\
& +\int_{\Gamma_{\text {in }} \cup \Gamma_{\text {out }}} \int_{\Xi} \frac{1}{\rho[f]}\left\{\frac{\partial a}{\partial \rho} \rho[f]+\frac{\partial a}{\partial \boldsymbol{v}} \cdot\{\boldsymbol{\xi}-\boldsymbol{u}(\rho[f], \boldsymbol{v}[f])\}\right\} \eta \mathrm{d} \boldsymbol{\xi} \mathrm{d} \Gamma .
\end{aligned}
$$

Next, we consider the derivation of $\delta_{f} E[f, g, \phi]$ in Eq. (47). We now consider the variation of $\delta_{f} G, \delta_{f} I$, and $\delta_{f} B_{n}$ individually, since $E=G+I+B_{n}$. Therefore, using the above derivation of $\delta_{f} J$ as a basis, we obtain the following variations:

$$
\begin{aligned}
\delta_{f} G= & {\left[\int_{D} \int_{\Xi} g \eta \mathrm{d} \xi \mathrm{d} \Omega\right]_{t_{0}}^{t_{1}}-\int_{I} \int_{D} \int_{\Xi} \frac{\partial g}{\partial t} \eta \mathrm{d} \xi \mathrm{d} \Omega \mathrm{d} t+\int_{I} \int_{\Gamma} \int_{\Xi} g \xi \cdot n \eta \mathrm{d} \xi \mathrm{d} \Gamma \mathrm{d} t } \\
& -\int_{I} \int_{D} \int_{\Xi} \xi \cdot \nabla g \eta \mathrm{d} \xi \mathrm{d} \Omega \mathrm{d} t+\int_{I} \int_{D} \int_{\Xi} \frac{1}{\tau_{\mathrm{B}}}\left(g-g^{\mathrm{eq}}\right) \eta \mathrm{d} \xi \mathrm{d} \Omega \mathrm{d} t, \\
\delta_{f} I= & \left.\int_{D} \int_{\Xi} g\right|_{t=t_{0}} \eta \mathrm{d} \xi \mathrm{d} \Omega, \\
\delta_{f} B_{1}= & \int_{I} \int_{\Gamma_{\mathrm{w}}} \int_{\Xi_{n \cdot \xi<0}} g\{\eta(\xi)-\eta(-\xi)\} \mathrm{d} \xi \mathrm{d} \Gamma \mathrm{d} t, \\
\delta_{f} B_{2}= & \int_{I} \int_{\Gamma_{\text {in }}} \int_{\Xi}\left(g-g_{\text {in }}^{\mathrm{eq}}\right) \eta \mathrm{d} \xi \mathrm{d} \Gamma \mathrm{d} t, \\
\delta_{f} B_{3}= & \int_{I} \int_{\Gamma_{\text {out }}} \int_{\Xi}\left(g-g_{\text {out }}^{\mathrm{eq}}\right) \eta \mathrm{d} \xi \mathrm{d} \Gamma \mathrm{d} t,
\end{aligned}
$$

where $g^{\mathrm{eq}}$ is the variation of $\int_{I} \int_{D} \int_{\Xi} f^{\mathrm{eq}} g \mathrm{~d} \xi \mathrm{d} \Omega \mathrm{d} t$ with respect to $f$, and can be obtained as follows:

$$
\begin{aligned}
& \int_{I} \int_{D} \int_{\Xi} f^{\mathrm{eq}}[f+\varepsilon \eta] g \mathrm{~d} \xi \mathrm{d} \Omega \mathrm{d} t \\
& =\int_{I} \int_{D} \int_{\Xi} f^{\mathrm{eq}}\{\rho[f+\varepsilon \eta], \boldsymbol{u}(\rho[f+\varepsilon \eta], \boldsymbol{v}[f+\varepsilon \eta])\} g \mathrm{~d} \xi \mathrm{d} \Omega \mathrm{d} t \\
& =\int_{I} \int_{D} \int_{\Xi} f^{\mathrm{eq}}\{\rho[f]+\varepsilon \rho[\eta], \boldsymbol{u}(\rho[f]+\varepsilon \rho[\eta], \boldsymbol{v}[f]+\varepsilon \boldsymbol{v}[\eta])\} g \mathrm{~d} \boldsymbol{\xi} \mathrm{d} \Omega \mathrm{d} t \\
& =\int_{I} \int_{D} \int_{\Xi} f^{\mathrm{eq}}\left\{\rho[f]+\varepsilon \rho[\eta], \boldsymbol{u}(\rho[f], \boldsymbol{v}[f])+\varepsilon \frac{\partial \boldsymbol{u}}{\partial \rho} \rho[\eta]+\varepsilon \frac{\partial \boldsymbol{u}}{\partial \boldsymbol{v}} \boldsymbol{v}[\eta]\right\} g \mathrm{~d} \boldsymbol{\xi} \mathrm{d} \Omega \mathrm{d} t+o(\varepsilon) \\
& =\int_{I} \int_{D} \int_{\Xi}\left\{f^{\mathrm{eq}}\{\rho[f], \boldsymbol{u}(\rho[f], \boldsymbol{v}[f])\}\right. \\
& \left.+\varepsilon \frac{\partial f^{\text {eq }}}{\partial \rho} \rho[\eta]+\frac{\partial f^{\text {eq }}}{\partial \boldsymbol{u}} \cdot\left(\varepsilon \frac{\partial \boldsymbol{u}}{\partial \rho} \rho[\eta]+\varepsilon \frac{\partial \boldsymbol{u}}{\partial \boldsymbol{v}} v[\eta]\right)\right\} g \mathrm{~d} \xi \mathrm{d} \Omega \mathrm{d} t+o(\varepsilon),
\end{aligned}
$$


thus,

$$
\begin{aligned}
\int_{I} \int_{D} \int_{\Xi} g^{\mathrm{eq}} \eta \mathrm{d} \boldsymbol{\xi} \mathrm{d} \Omega \mathrm{d} t & =\int_{I} \int_{D} \int_{\Xi}\left\{\frac{\partial f^{\mathrm{eq}}}{\partial \rho} \rho[\eta]+\frac{\partial f^{\mathrm{eq}}}{\partial \boldsymbol{u}} \cdot\left(\frac{\partial \boldsymbol{u}}{\partial \rho} \rho[\eta]+\frac{\partial \boldsymbol{u}}{\partial \boldsymbol{v}} \boldsymbol{v}[\eta]\right)\right\} g \mathrm{~d} \hat{\boldsymbol{\xi}} \mathrm{d} \boldsymbol{\mathrm { d }} \Omega \mathrm{d} t \\
& =\int_{I} \int_{D} \int_{\Xi} \int_{\Xi}\left\{\frac{\partial f^{\mathrm{eq}}}{\partial \rho}+\frac{\partial f^{\mathrm{eq}}}{\partial \boldsymbol{u}} \cdot\left(\frac{\partial \boldsymbol{u}}{\partial \rho}+\frac{\partial \boldsymbol{u}}{\partial \boldsymbol{v}} \hat{\boldsymbol{\xi}}\right)\right\} g \eta(\hat{\boldsymbol{\xi}}) \mathrm{d} \hat{\boldsymbol{\xi}} \mathrm{d} \boldsymbol{\xi} \mathrm{d} \Omega \mathrm{d} t \\
& =\int_{I} \int_{D} \int_{\Xi} \int_{\Xi}\left\{\frac{1}{\rho} f^{\mathrm{eq}}+\frac{\boldsymbol{\xi}-\boldsymbol{u}}{T} f^{\mathrm{eq}} \cdot\left(-\frac{\boldsymbol{v}}{\rho^{2}}+\frac{1}{\rho} \hat{\boldsymbol{\xi}}\right)\right\} g \eta(\hat{\boldsymbol{\xi}}) \mathrm{d} \hat{\boldsymbol{\xi}} \mathrm{d} \boldsymbol{\xi} \mathrm{d} \Omega \mathrm{d} t \\
& =\int_{I} \int_{D} \int_{\Xi} \int_{\Xi} \frac{g f^{\mathrm{eq}}}{\rho T}\{T+(\boldsymbol{\xi}-\boldsymbol{u}) \cdot(\hat{\boldsymbol{\xi}}-\boldsymbol{u})\} \eta(\hat{\boldsymbol{\xi}}) \mathrm{d} \hat{\boldsymbol{\xi}} \mathrm{d} \boldsymbol{\xi} \mathrm{d} \Omega \mathrm{d} t .
\end{aligned}
$$

In addition, $g_{\text {in }}^{\text {eq }}$ and $g_{\text {out }}^{\mathrm{eq}}$ can be also obtained using the above formulation. Thus, we have

$$
\begin{aligned}
& g^{\mathrm{eq}}=\int_{\Xi} \frac{g(\hat{\boldsymbol{\xi}}) f^{\mathrm{eq}}}{\rho T}\{T+(\hat{\boldsymbol{\xi}}-\boldsymbol{u}) \cdot(\boldsymbol{\xi}-\boldsymbol{u})\} \mathrm{d} \hat{\boldsymbol{\xi}}, \\
& g_{\text {in }}^{\mathrm{eq}}=\int_{\Xi} \frac{g(\hat{\boldsymbol{\xi}}) f^{\mathrm{eq}}}{\rho} \mathrm{d} \hat{\boldsymbol{\xi}}, \\
& g_{\text {out }}^{\mathrm{eq}}=\int_{\Xi} \frac{g(\hat{\boldsymbol{\xi}}) f^{\mathrm{eq}}}{\rho T}(\hat{\boldsymbol{\xi}}-\boldsymbol{u}) \cdot(\boldsymbol{\xi}-\boldsymbol{u}) \mathrm{d} \hat{\boldsymbol{\xi}} .
\end{aligned}
$$

We now derive the adjoint equation and its boundary and initial conditions, using the Eqs. (B.16)-(B.21). First, the adjoint equation is obtained from Eq. (B.17), ignoring first and third terms, as

$$
\int_{I} \int_{D} \int_{\Xi}\left\{-\frac{\partial g}{\partial t}-\xi \cdot \nabla g+\frac{1}{\tau_{\mathrm{B}}}\left(g-g^{\mathrm{eq}}\right)\right\} \eta \mathrm{d} \xi \mathrm{d} \Omega \mathrm{d} t=0 .
$$

Since $\eta$ represents any function, the adjoint equation is defined as the integrand of Eq. (B.27):

$$
-\frac{\partial g}{\partial t}-\xi \cdot \nabla g+\frac{1}{\tau_{\mathrm{B}}}\left(g-g^{\mathrm{eq}}\right)=0 \quad \text { in } \quad I \times D \times \Xi .
$$

Next, we consider the initial condition of the adjoint equation. From Eqs. (B.16), (B.17) and (B.18), we obtain the following equation:

$$
\begin{aligned}
\int_{D} \int_{\Xi} A^{\prime} \eta\left(t_{1}\right) \mathrm{d} \xi \mathrm{d} \Omega+\int_{\Gamma_{\text {in }} \cup \Gamma_{\text {out }}} \int_{\Xi} a^{\prime} \eta\left(t_{1}\right) \mathrm{d} \xi \mathrm{d} \Omega \\
\quad+\int_{D} \int_{\Xi}\left\{g\left(t_{1}\right) \eta\left(t_{1}\right)-g\left(t_{0}\right) \eta\left(t_{0}\right)\right\} \mathrm{d} \xi \mathrm{d} \Omega+\int_{D} \int_{\Xi} g\left(t_{0}\right) \eta\left(t_{0}\right) \mathrm{d} \xi \mathrm{d} \Omega=0,
\end{aligned}
$$

where

$$
\begin{aligned}
& A^{\prime}=\frac{1}{\rho}\left\{\frac{\partial A}{\partial \rho} \rho+\frac{\partial A}{\partial \boldsymbol{u}} \cdot(\boldsymbol{\xi}-\boldsymbol{u})\right\} \\
& a^{\prime}=\frac{1}{\rho}\left\{\frac{\partial a}{\partial \rho} \rho+\frac{\partial a}{\partial \boldsymbol{u}} \cdot(\xi-\boldsymbol{u})\right\} .
\end{aligned}
$$

Therefore, the initial condition of the adjoint equation is the following,

$$
g\left(t_{1}\right)+A^{\prime}=0 \text { in } D \times \Xi .
$$

We note that the second term of Eq. (B.29) affects the initial boundary conditions shown in Eqs. (50) and (51). 
Finally, we consider the boundary conditions of the adjoint equation. From the third term of Eq. (B.16), and Eqs. (B.19)-(B.21), we obtain the following:

$$
\begin{aligned}
\int_{I} \int_{\Gamma} \int_{\Xi} g \boldsymbol{\xi} & \cdot \boldsymbol{n} \eta \mathrm{d} \boldsymbol{\xi} \mathrm{d} \Gamma \mathrm{d} t+\int_{I} \int_{\Gamma_{\mathrm{w}}} \int_{\Xi_{\boldsymbol{n}} \boldsymbol{\xi}<0} g\{\eta(\boldsymbol{\xi})-\eta(-\boldsymbol{\xi})\} \mathrm{d} \boldsymbol{\xi} \mathrm{d} \Gamma \mathrm{d} t \\
& +\int_{I} \int_{\Gamma_{\text {in }}} \int_{\Xi}\left(g-g_{\text {in }}^{\mathrm{eq}}\right) \eta \mathrm{d} \boldsymbol{\xi} \mathrm{d} \Gamma \mathrm{d} t+\int_{I} \int_{\Gamma_{\text {out }}} \int_{\Xi}\left(g-g_{\text {out }}^{\mathrm{eq}}\right) \eta \mathrm{d} \boldsymbol{\xi} \mathrm{d} \Gamma \mathrm{d} t=0 .
\end{aligned}
$$

Using the assumption of $\eta$ in (B.2), the second term of the above equation can be eliminated. Therefore, the above equation is rewritten as

$$
\begin{array}{r}
\int_{I} \int_{\Gamma_{\mathrm{w}}} \int_{\Xi} g \boldsymbol{\xi} \cdot \boldsymbol{n} \eta \mathrm{d} \boldsymbol{\xi} \mathrm{d} \Gamma \mathrm{d} t+\int_{I} \int_{\Gamma_{\text {in }}} \int_{\Xi} g \boldsymbol{\xi} \cdot \boldsymbol{n} \eta \mathrm{d} \boldsymbol{\xi} \mathrm{d} \Gamma \mathrm{d} t+\int_{I} \int_{\Gamma_{\text {out }}} \int_{\Xi} g \boldsymbol{\xi} \cdot \boldsymbol{n} \eta \mathrm{d} \boldsymbol{\xi} \mathrm{d} \Gamma \mathrm{d} t \\
+\int_{I} \int_{\Gamma_{\text {in }}} \int_{\Xi}\left(g-g_{\text {in }}^{\mathrm{eq}}\right) \eta \mathrm{d} \boldsymbol{\xi} \mathrm{d} \Gamma \mathrm{d} t+\int_{I} \int_{\Gamma_{\text {out }}} \int_{\Xi}\left(g-g_{\text {out }}^{\mathrm{eq}}\right) \eta \mathrm{d} \boldsymbol{\xi} \mathrm{d} \Gamma \mathrm{d} t=0 .
\end{array}
$$

Here, using the first term of the above equation, the boundary condition of $\Gamma_{\mathrm{w}}$ is obtained as follows:

$$
\begin{aligned}
\int_{I} \int_{\Gamma_{\mathrm{w}}} \int_{\Xi} g \boldsymbol{\xi} \cdot \boldsymbol{n} \eta \mathrm{d} \boldsymbol{\xi} \mathrm{d} \Gamma \mathrm{d} t & =\int_{I} \int_{\Gamma_{\mathrm{w}}} \int_{\Xi_{n} \cdot \xi \geqslant 0} g(\boldsymbol{\xi}) \boldsymbol{\xi} \cdot \boldsymbol{n} \eta \mathrm{d} \boldsymbol{\xi} \mathrm{d} \Gamma \mathrm{d} t+\int_{I} \int_{\Gamma_{\mathrm{w}}} \int_{\Xi_{n} \cdot \boldsymbol{\xi}<0} g(\boldsymbol{\xi}) \boldsymbol{\xi} \cdot \boldsymbol{n} \eta \mathrm{d} \boldsymbol{\xi} \mathrm{d} \Gamma \mathrm{d} t \\
& =\int_{I} \int_{\Gamma_{\mathrm{w}}} \int_{\Xi_{\boldsymbol{n}} \cdot \boldsymbol{\xi} \geqslant 0} g(\boldsymbol{\xi}) \boldsymbol{\xi} \cdot \boldsymbol{n} \eta \mathrm{d} \boldsymbol{\xi} \mathrm{d} \Gamma \mathrm{d} t-\int_{I} \int_{\Gamma_{\mathrm{w}}} \int_{\Xi_{\boldsymbol{n}} \cdot \xi \geqslant 0} g(-\boldsymbol{\xi}) \boldsymbol{\xi} \cdot \boldsymbol{n} \eta \mathrm{d} \boldsymbol{\xi} \mathrm{d} \Gamma \mathrm{d} t \\
& =\int_{I} \int_{\Gamma_{\mathrm{w}}} \int_{\Xi_{n} \cdot \boldsymbol{\xi} \geqslant 0} \boldsymbol{\xi} \cdot \boldsymbol{n}\{g(\boldsymbol{\xi})-g(-\boldsymbol{\xi})\} \eta \mathrm{d} \boldsymbol{\xi} \mathrm{d} \Gamma \mathrm{d} t \\
& =\int_{I} \int_{\Gamma_{\mathrm{w}}} \int_{\Xi_{\boldsymbol{n} \cdot \boldsymbol{\xi}>0}} \boldsymbol{\xi} \cdot \boldsymbol{n}\{g(\boldsymbol{\xi})-g(-\boldsymbol{\xi})\} \eta \mathrm{d} \boldsymbol{\xi} \mathrm{d} \Gamma \mathrm{d} t
\end{aligned}
$$

where $\Xi_{n \cdot \boldsymbol{\xi}>0}$ is the velocity space of $\boldsymbol{\xi}$ that satisfies $\boldsymbol{n} \cdot \boldsymbol{\xi}>0$. We note that the boundary condition of $\Gamma_{\mathrm{w}}$ for the adjoint equation has to be set in $\boldsymbol{n} \cdot \boldsymbol{\xi}>0$, while the boundary condition of Boltzmann equation has to be set so that $\boldsymbol{n} \cdot \boldsymbol{\xi}<0$. The reason why different settings are required for the Boltzmann equation and the adjoint equation is that both the propagation and time evolution directions of adjoint equation are opposite those of the Boltzmann equation. Consequently, from the above equation, we obtain the following boundary condition:

$$
g(\boldsymbol{\xi})-g(-\boldsymbol{\xi})=0 \quad \text { in } \quad I \times \Gamma_{\mathrm{w}} \times \Xi_{\boldsymbol{n} \cdot \boldsymbol{\xi}>0} .
$$

Similarly, the boundary conditions for $\Gamma_{\text {in }}$ and $\Gamma_{\text {out }}$ can be described as follows:

$$
\begin{array}{lll}
g-g_{\text {in }}^{\text {eq }}=0 & \text { in } & I \times \Gamma_{\text {in }} \times \Xi, \\
g-g_{\text {out }}^{\text {eq }}=0 & \text { in } & I \times \Gamma_{\text {out }} \times \Xi .
\end{array}
$$

\section{References}

[1] O. Pironneau, On optimum profiles in Stokes flow, Journal of Fluid Mechanics 59 (1) (1973) 117-128.

[2] O. Pironneau, On optimum design in fluid mechanics, Journal of Fluid Mechanics 64 (1) (1974) 97-110.

[3] B. Mohammadi, O. Pironneau, Applied shape optimization for fluid, Oxford University Press, Oxford, 2001.

[4] E. Katamine, T. Tsubata, H. Azegami, Solution to shape optimization problem of viscous flow fields considering convection term, Inverse Problems in Engineering Mechanics 4 (2003) 401-408.

[5] B. Mohammadi, O. Pironneau, Shape optimization in fluid mechanics, Annual Review of Fluid Mechanics 36 (2004) $255-279$.

[6] A. Wang, Y. Ma, Z. Gao, Shape optimization of a body in the Oseen flow, Numerical Methods for Partial Differential Equations 26 (6) (2009) 1642-1659.

[7] Y. Iwata, H. Azegami, T. Aoyama, E. Katamine, Numerical solution to shape optimization problems for non-stationary Navier-Stokes problems, JSIAM Letters 2 (2010) 733-740.

[8] L. Wang, Y. Fan, L. Luo, Heuristic optimality criterion algorithm for shape design of fluid flow, Journal of Computational Physics 229 (20) (2010) 8031-8044.

[9] D. N. Srinath, S. Mittal, An adjoint method for shape optimization in unsteady viscous flows, Journal of Computational Physics 229 (6) (2010) 1994-2008. 
[10] A. S. Zymaris, D. I. Papadimitriou, K. C. Giannakoglou, C. Othmer, Adjoint wall functions: A new concept for use in aerodynamic shape optimization, Journal of Computational Physics 229 (13) (2010) 5228-5245.

[11] M. Zabarankin, A. Molyboha, 3D shape optimization in viscous incompressible fluid under Oseen approximation, SIAM Journal on Control and Optimization 49 (3) (2011) 1358-1382.

[12] M. P. Bendsøe, N. Kikuchi, Generating optimal topologies in structural design using a homogenization method, Computer Methods in Applied Mechanics and Engineering 71 (2) (1988) 197-224.

[13] K. Suzuki, N. Kikuchi, A homogenization method for shape and topology optimization, Computer Methods in Applied Mechanics and Engineering 93 (3) (1991) 291-318.

[14] A. R. Diaz, N. Kikuchi, Solutions to shape and topology eigenvalue optimization problems using a homogenization method, International Journal for Numerical Methods in Engineering 35 (7) (1992) 1487-1502.

[15] Z.-D. Ma, N. Kikuchi, H.-C. Cheng, Topological design for vibrating structures, Computer Methods in Applied Mechanics and Engineering 121 (1) (1995) 259-280.

[16] N. L. Pedersen, Maximization of eigenvalues using topology optimization, Structural and Multidisciplinary Optimization 20 (1) (2000) $2-11$.

[17] Q. Li, G. P. Steven, O. M. Querin, Y. Xie, Shape and topology design for heat conduction by evolutionary structural optimization, International Journal of Heat and Mass Transfer 42 (17) (1999) 3361-3371.

[18] A. Iga, S. Nishiwaki, K. Izui, M. Yoshimura, Topology optimization for thermal conductors considering design-dependent effects, including heat conduction and convection, International Journal of Heat and Mass Transfer 52 (11) (2009) 2721-2732.

[19] T. Borrvall, J. Petersson, Topology optimization of fluids in Stokes flow, International Journal for Numerical Methods in Fluids 41 (1) (2003) 77-107.

[20] L. H. Olesen, F. Okkels, H. Bruus, A high-level programming-language implementation of topology optimization applied to steady-state Navier-Stokes flow, International Journal for Numerical Methods in Engineering 65 (7) (2006) 975-1001

[21] F. Murat, L. Tartar, Optimality conditions and homogenization, Research Notes in Mathematics 127 (1985) 1-8.

[22] M. P. Bendsøe, O. Sigmund, Topology optimization: theory, methods and applications, Springer, New York, 2003.

[23] G. Allaire, F. Jouve, A.-M. Toader, A level-set method for shape optimization, Comptes Rendus Mathematique 334 (12) (2002) 1125-1130.

[24] M. Y. Wang, X. Wang, D. Guo, A level set method for structural topology optimization, Computer Methods in Applied Mechanics and Engineering 192 (1) (2003) 227-246.

[25] G. Allaire, F. Jouve, A.-M. Toader, Structural optimization using sensitivity analysis and a level-set method, Journal of Computational Physics 194 (1) (2004) 363-393.

[26] B. Bourdin, A. Chambolle, Topology optimization approaches, ESAIM: Control, Optimisation and Calculus of Variations 9 (2003) 1948.

[27] A. Takezawa, S. Nishiwaki, M. Kitamura, Shape and topology optimization based on the phase field method and sensitivity analysis, Journal of Computational Physics 229 (7) (2010) 2697-2718.

[28] A. Takezawa, M. Kitamura, Phase field method to optimize dielectric devices for electromagnetic wave propagation, Journal of Computational Physics 257 (2014) 216-240.

[29] Y. Xie, G. P. Steven, A simple evolutionary procedure for structural optimization, Computers and structures 49 (5) (1993) 885-896.

[30] O. Sigmund, K. Maute, Topology optimization approaches, Structural and Multidisciplinary Optimization 48 (6) (2013) 1031-1055.

[31] N. Aage, T. H. Poulsen, A. Gersborg-Hansen, O. Sigmund, Topology optimization of large scale Stokes flow problems, Structural and Multidisciplinary Optimization 35 (2) (2008) 175-180.

[32] Y. Deng, Z. Liu, P. Zhang, Y. Liu, Y. Wu, Topology optimization of unsteady incompressible Navier-Stokes flows, Journal of Computational Physics 230 (17) (2011) 6688-6708.

[33] S. Kreissl, K. Maute, Levelset based fluid topology optimization using the extended finite element method, Structural and Multidisciplinary Optimization 46 (3) (2012) 311-326.

[34] T. Kondoh, T. Matsumori, A. Kawamoto, Drag minimization and lift maximization in laminar flows via topology optimization employing simple objective function expressions based on body force integration, Structural and Multidisciplinary Optimization 45 (5) (2011) 693-701.

[35] A. Gersborg-Hansen, O. Sigmund, R. B. Haber, Topology optimization of channel flow problems, Structural and Multidisciplinary Optimization 30 (3) (2005) 181-192.

[36] C. S. Andreasen, A. R. Gersborg, O. Sigmund, Topology optimization of microfluidic mixers, International Journal for Numerical Methods in Fluids 61 (5) (2009) 498-513.

[37] Z. Liu, Q. Gao, P. Zhang, M. Xuan, Y. Wu, Topology optimization of fluid channels with flow rate equality constraints, Structural and Multidisciplinary Optimization 44 (1) (2010) 31-37.

[38] G. H. Yoon, Topology optimization for stationary fluid-structure interaction problems using a new monolithic formulation, International Journal for Numerical Methods in Engineering 82 (5) (2010) 591-616.

[39] M. M. Gregersen, F. Okkels, M. Z. Bazant, H. Bruus, Topology and shape optimization of induced-charge electro-osmotic micropumps, New Journal of Physics 11 (7) (2009) 075019.

[40] E. Papoutsis-Kiachagias, E. Kontoleontos, A. Zymaris, D. Papadimitriou, K. Giannakoglou, Constrained topology optimization for laminar and turbulent flows, including heat transfer, CIRA, editor, EUROGEN, Evolutionary and Deterministic Methods for Design, Optimization and Control, Capua, Italy, 2011.

[41] T. Matsumori, T. Kondoh, A. Kawamoto, T. Nomura, Topology optimization for fluid-thermal interaction problems under constant input power, Structural and Multidisciplinary Optimization 47 (4) (2013) 571-581.

[42] G. H. Yoon, Topological layout design of electro-fluid-thermal-compliant actuator, Computer Methods in Applied Mechanics and Engineering 209-212 (2012) 28-44.

[43] J. K. Guest, J. H. Prévost, Topology optimization of creeping fluid flows using a Darcy-Stokes finite element, International Journal for Numerical Methods in Engineering 66 (3) (2006) 461-484.

[44] J. A. Sethian, A. Wiegmann, Structural boundary design via level set and immersed interface methods, Journal of Computational Physics 163 (2) (2000) 489-528. 
[45] S. J. Osher, F. Santosa, Level set methods for optimization problems involving geometry and constraints, Journal of Computational Physics 171 (1) (2001) 272-288.

[46] A. Leitao, O. scherzer, On the relation between constraint regularization, level sets and shape optimization, Inverse Problems 19 (1) (2003) L1-L11.

[47] S. Amstutz, H. Andrä, A new algorithm for topology optimization using a level-set method, Journal of Computational Physics 216 (2) (2006) $573-588$.

[48] S. Osher, J. A. Sethian, Front propagating with curvature dependent speed: algorithms based on hamilton-jacobi formulations, Journal of Computational Physics 79 (1988) 12-49.

[49] H.-K. Zhao, T. Chan, B. Merriman, S. Osher, A variational level set approach to multiphase motion, Journal of computational physics 127 (1) (1996) 179-195.

[50] V. J. Challis, J. K. Guest, Level set topology optimization of fluids in Stokes flow, International Journal for Numerical Methods in Engineering 79 (10) (2009) 1284-1308.

[51] X. Duan, Y. Ma, R. Zhang, Optimal shape control of fluid flow using variational level set method, Physics Letters A 372 (9) (2008) 13741379.

[52] S. Zhou, Q. Li, A variational level set method for the topology optimization of steady-state Navier-Stokes flow, Journal of Computational Physics 227 (24) (2008) 10178-10195.

[53] X.-B. Duan, Y.-C. Ma, R. Zhang, Shape-topology optimization for Navier-Stokes problem using variational level set method, Journal of Computational and Applied Mathematics 222 (2) (2008) 487-499.

[54] Y. Deng, P. Zhang, Y. Liu, Y. Wu, Z. Liu, Optimization of unsteady incompressible Navier-Stokes flows using variational level set method, International Journal for Numerical Methods in Fluids 71 (12) (2013) 1475-1493.

[55] G. Allaire, F. Gournay, F. Jouve, A.-M. Toader, Structural optimization using topological and shape sensitivity via a level set method, Internal Report 555, Ecole Polytechnique, France, 2004.

[56] H. A. Eschenauer, V. V. Kobelev, A. Schumacher, Bubble method for topology and shape optimization of structures, Structural Optimization 8 (1) (1994) 42-51.

[57] J. Sokolowski, A. Zochowski, On the topological derivative in shape optimization, SIAM Journal on Control and Optimization 37 (4) (1999) $1251-1272$.

[58] L. He, C.-Y. Kao, S. Osher, Incorporating topological derivatives into shape derivatives based level set methods, Journal of Computational Physics 225 (1) (2007) 891-909.

[59] T. Yamada, K. Izui, S. Nishiwaki, A. Takezawa, A topology optimization method based on the level set method incorporating a fictitious interface energy, Computer Methods in Applied Mechanics and Engineering 199 (45-48) (2010) 2876-2891.

[60] M. Otomori, T. Yamada, K. Izui, S. Nishiwaki, Level set-based topology optimisation of a compliant mechanism design using mathematical programming, Mechanical Sciences 2 (1) (2011) 91-98.

[61] T. Yamada, S. Yamasaki, S. Nishiwaki, K. Izui, M. Yoshimura, Design of compliant thermal actuators using structural optimization based on the level set method, Journal of Computing and Information Science in Engineering 11 (1) (2011) 011005.

[62] T. Yamada, K. Izui, S. Nishiwaki, A level set-based topology optimization method for maximizing thermal diffusivity in problems including design-dependent effects, Journal of Mechanical Design 133 (3) (2011) 031011.

[63] M. Otomori, T. Yamada, K. Izui, S. Nishiwaki, J. A. Andkjær, A topology optimization method based on the level set method for the design of negative permeability dielectric metamaterials, Computer Methods in Applied Mechanics and Engineering 237-240 (2012) 192-211.

[64] M. Otomori, T. Yamada, J. A. Andkjær, K. Izui, S. Nishiwaki, N. Kogiso, Level set-based topology optimization for the design of an electromagnetic cloak with ferrite material, IEEE Transactions on Magnetics 49 (5) (2013) 2081-2084.

[65] G. Fujii, H. Watanabe, T. Yamada, T. Ueta, M. Mizuno, Level set based topology optimization for optical cloaks, Applied Physics Letters $102(25)(2013) 251106$.

[66] T. Yamada, H. Watanabe, G. Fujii, T. Matsumoto, Topology optimization for a dielectric optical cloak based on an exact level set approach, IEEE Transactions on Magnetics 49 (5) (2013) 2073-2076.

[67] L. Lu, T. Yamamoto, M. Otomori, T. Yamada, K. Izui, S. Nishiwaki, Topology optimization of an acoustic metamaterial with negative bulk modulus using local resonance, Finite Elements in Analysis and Design 72 (2013) 1-12.

[68] C. Talischi, G. H. Paulino, An operator splitting algorithm for Tikhonov-regularized topology optimization, Computer Methods in Applied Mechanics and Engineering 253 (2013) 599-608.

[69] G. R. McNamura, G. Zanetti, Use of the Boltzmann equation to simulate lattice-gas automata, Physical Review Letters 61 (20) (1988) $2332-2335$

[70] F. Higuera, S. Succi, R. Benzi, Lattice gas dynamics with enhanced collisions, Europhysics Letters 9 (4) (1989) 345-349.

[71] R. Benzi, S. Succi, M. Vergassola, The lattice Boltzmann equation: theory and applications, Physics Reports 222 (3) (1992) $145-197$.

[72] S. Chen, G. D. Doolen, Lattice Boltzmann method for fluid flows, Annual Review of Fluid Mechanics 30 (1) (1998) 329-364.

[73] S. Succi, Lattice Boltzmann equation for fluid dynamics and beyond, Oxford University Press, New York, 2001.

[74] C. K. Aidun, J. R. Clausen, Lattice-Boltzmann method for complex flows, Annual Review of Fluid Mechanics 42 (1) (2010) $439-472$

[75] T. Inamuro, M. Yoshino, F. Ogino, Accuracy of the lattice Boltzmann method for small Knudsen number with finite Reynolds number, Physics of Fluids 9 (11) (1997) 3535-3542.

[76] T. Inamuro, M. Yoshino, H. Inoue, R. Mizuno, F. Ogino, A lattice Boltzmann method for a binary miscible fluid mixture and its application to a heat-transfer problem, Journal of Computational Physics 179 (1) (2002) 201-215.

[77] X. He, L.-S. Luo, A priori derivation of the lattice Boltzmann equation, Physical Review E 55 (6) (1997) R6333-R6336.

[78] M. Junk, A. Klar, L.-S. Luo, Asymptotic analysis of the lattice Boltzmann equation, Journal of Computational Physics 210 (2) (2005) 676-704.

[79] X. Shan, H. Chen, Lattice Boltzmann model for simulating flows with multiple phases and components, Physical Review E 47 (3) (1993) $1815-1819$.

[80] T. Inamuro, T. Ogata, S. Tajima, N. Konishi, A lattice Boltzmann method for incompressible two-phase flows with large density differences, 
Journal of Computational Physics 198 (2) (2004) 628-644.

[81] M. Yoshino, Y. Mizutani, Lattice Boltzmann simulation of liquid-gas flows through solid bodies in a square duct, Mathematics and Computers in Simulation 72 (2) (2006) 264-269.

[82] T. Inamuro, Lattice Boltzmann methods for viscous fluid flows and for two-phase fluid flows, Fluid Dynamics Research 38 (9) (2006) 641-659.

[83] S. Succi, E. Foti, F. Higuera, Three-dimensional flows in complex geometries with the lattice Boltzmann method, Europhysics Letters 10 (5) (1989) 433-438.

[84] M. A. A. Spaid, F. R. Phelan, Lattice Boltzmann methods for modeling microscale flow in fibrous porous media, Physics of Fluids 9 (9) (1997) 2468-2474.

[85] Z. Guo, T. Zhao, Lattice Boltzmann model for incompressible flows through porous media, Physical Review E 66 (3) (2002) 036304.

[86] M. Yoshino, T. Inamuro, Lattice Boltzmann simulations for flow and heat/mass transfer problems in a three-dimensional porous structure, International Journal for Numerical Methods in Fluids 43 (2) (2003) 183-198.

[87] M. Briscolini, P. Santangelo, S. Succi, R. Benzi, Extended self-similarity in the numerical simulation of three-dimensional homogeneous flows, Physical Review E 50 (3) (1994) R1745-R1747.

[88] S. Hou, Q. Zou, S. Chen, G. Doolen, A. C. Cogley, Simulation of cavity flow by the lattice Boltzmann method, Journal of Computational Physics 118 (2) (1995) 329-347.

[89] H. Chen, S. Kandasamy, S. Orszag, R. Shock, S. Succi, V. Yakhot, Extended Boltzmann kinetic equation for turbulent flows, Science $301(5633)(2003) 633-636$

[90] L.-S. Lin, Y.-C. Chen, C.-A. Lin, Multi relaxation time lattice boltzmann simulations of deep lid driven cavity flows at different aspect ratios, Computers and Fluids 45 (1) (2011) 233-240.

[91] S. S. Chikatamarla, I. V. Karlin, Entropic lattice Boltzmann method for turbulent flow simulations: Boundary conditions, Physica A: Statistical Mechanics and its Applications 392 (9) (2013) 1925-1930.

[92] Z.-G. Feng, E. E. Michaelides, The immersed boundary-lattice Boltzmann method for solving fluid-particles interaction problems, Journal of Computational Physics 195 (2) (2004) 602-628

[93] C. Shu, N. Liu, Y. Chew, A novel immersed boundary velocity correction-lattice Boltzmann method and its application to simulate flow past a circular cylinder, Journal of Computational Physics 226 (2) (2007) 1607-1622.

[94] K. Suzuki, T. Inamuro, Effect of internal mass in the simulation of a moving body by the immersed boundary method, Computers and Fluids 49 (1) (2011) 173-187.

[95] K. Suzuki, T. Inamuro, A higher-order immersed boundary-lattice Boltzmann method using a smooth velocity field near boundaries, Computers and Fluids 76 (2013) 105-115.

[96] G. Pingen, A. Evgrafov, K. Maute, Topology optimization of flow domains using the lattice Boltzmann method, Structural and Multidisciplinary Optimization 34 (6) (2007) 507-524.

[97] G. Pingen, M. Waidmann, A. Evgrafov, K. Maute, A parametric level-set approach for topology optimization of flow domains, Structural and Multidisciplinary Optimization 41 (1) (2009) 117-131.

[98] S. Kreissl, G. Pingen, K. Maute, An explicit level set approach for generalized shape optimization of fluids with the lattice Boltzmann method, International Journal for Numerical Methods in Fluids 65 (5) (2011) 496-519.

[99] G. Pingen, K. Maute, Optimal design for non-Newtonian flows using a topology optimization approach, Computers and Mathematics with Applications 59 (7) (2010) 2340-2350.

[100] S. Kreissl, G. Pingen, A. Evgrafov, K. Maute, Topology optimization of flexible micro-fluidic devices, Structural and Multidisciplinary Optimization 42 (4) (2010) 495-516

[101] D. Makhija, G. Pingen, R. Yang, K. Maute, Topology optimization of multi-component flows using a multi-relaxation time lattice Boltzmann method, Computers and Fluids 67 (2012) 104-114.

[102] G. Pingen, A. Evgrafov, K. Maute, A parallel Schur complement solver for the solution of the adjoint steady-state lattice Boltzmann equations: application to design optimisation, International Journal of Computational Fluid Dynamics 22 (7) (2008) 457-464.

[103] M. J. Krause, G. Thäter, V. Heuveline, Adjoint-based fluid flow control and optimisation with lattice Boltzmann methods, Computers and Mathematics with Applications 65 (6) (2013) 945-960.

[104] P. L. Bhatnagar, E. P. Gross, M. Krook, A model for collision processes in gases. I. Small amplitude processes in charged and neutral one-component systems, Physical Review 94 (3) (1954) 511-525.

[105] H. Cabannes, The discrete Boltzmann equation, theory and applications, University of California Press, California, 1980.

[106] R. M. Errico, What is an adjoint model?, Bulletin of the American Meteorological Society 78 (11) (1997) 2577-2592.

[107] T. Belytschko, S. Xiao, C. Parimi, Topology optimization with implicit functions and regularization, International Journal for Numerical Methods in Engineering 57 (8) (2003) 1177-1196.

[108] J. Norato, R. Haber, D. Tortorelli, M. P. Bendsøe, A geometry projection method for shape optimization, International Journal for Numerical Methods in Engineering 60 (14) (2004) 2289-2312.

[109] E. Sanchez-Palencia, Non-homogeneous media and vibration theory, Springer, Berlin, 1980.

[110] Y. Deng, Z. Liu, Y. Wu, Topology optimization of steady and unsteady incompressible Navier-Stokes flows driven by body forces, Structural and Multidisciplinary Optimization 47 (4) (2012) 555-570.

[111] A. N. Tikhonov, Numerical methods for the solution of ill-posed problems, Kluwer Academic Publishers, Boston, 1995.

[112] G. Pingen, A. Evgrafov, K. Maute, Adjoint parameter sensitivity analysis for the hydrodynamic lattice Boltzmann method with applications to design optimization, Computers and Fluids 38 (4) (2009) 910-923.

[113] M. J. Krause, V. Heuveline, Parallel fluid flow control and optimisation with lattice Boltzmann methods and automatic differentiation, Computers and Fluids 80 (2013) 28-36.

[114] R. T. Rockafellar, The multiplier method of Hestenes and Powell applied to convex programming, Journal of Optimization Theory and Applications 12 (6) (1973) 555-562.

[115] K. Yaji, T. Yamada, K. Izui, S. Nishiwaki, Topology optimization method using level set boundary expressions in Navier-Stokes flow, in: 
proceedings of 14th AIAA/ISSMO Multidisciplinary Analysis and Optimization Conference (2012MAO), Indiana, 2012.

[116] S. Whitaker, Flow in porous media I: A theoretical derivation of Darcy's law, Transport in Porous Media 1 (1) (1986) 3-25. 